DOI https://doi.org/10.24297/jam.v19i.9030

\title{
Spin-Valued Four Bosons Electrodynamics
}

\author{
R. Doria ${ }^{1}$, I. Soares ${ }^{2}$ \\ ${ }^{1}$ Aprendanet, Petrópolis, Brazil,Quarks, Petrópolis, Brazil \\ ${ }^{2}$ CBPF, Rio de Janeiro, Brazil, Quarks Petrópolis, Brazil \\ ${ }^{1}$ doria@aprendanet.com.br, ${ }^{2}$ w.inacio@hotmail.com
}

\begin{abstract}
Electromagnetism is based on electric charge and spin. The study here corresponds to understand on spin effects at a vectorial electrodynamics. Its scenario is a non-linear abelian electromagnetism where the electric charge is transmitted through a four bosons quadruplet, constituted by the usual photon, massive photon and charged massive photons. These four bosons intermediate the charge exchange $\Delta Q=0, \pm 1$.
\end{abstract}

The spin is introduced at first principles. A spintronics Lagrangian for four vector fields is performed. Considering that spin is a space-time physical entity derived from Lorentz Group, these vector fields are associated to Lorentz Group, as Lie algebra valued. Similarly to non-abelian gauge theories where $A_{\mu} \equiv A_{\mu a} t_{a}$, one introduces the relationship $A_{\mu} \equiv A_{\mu, \kappa \lambda} \Sigma^{\kappa \lambda}$ where $\left(\Sigma^{\kappa \lambda}\right)_{\alpha \beta}$ is the Lorentz Group generator.

Thus, based on three fundamentals which are light invariance, electric charge conservation law and vector fields Lie algebra valued through Lorentz Group generators, one derives a spin-valued four vectorial electrodynamics. It is given by the fields quadruplet $A_{\mu_{I}} \equiv\left\{A_{\mu}, U_{\mu}, V_{\mu}^{ \pm}\right\}$where $A_{\mu}$ means the usual photon, $U_{\mu}$ a massive photon and $V_{\mu}^{ \pm}$ massive charged photons. Two novelties appear. The first one is that, new terms are developed into usual four bosons electromagnetism. They contribute to Lagrangian, equations of motion, Noether theorem. The second one is that the equations of motion derive a renormalizable spin coupling with the electric and magnetic fields.

There is a spin-1 electrodynamics to be investigated. A neutral electromagnetism is mandatory to be analyzed. Something beyond dipole, quadrupole and so on. Understand the role of spin in the electrical and magnetic properties of particles. A spin vectorial expression $\vec{S}$ is obtained. It adds EM interactions not depending on electric charge and with spin interactions through electric dipole and magnetic moments.

\section{Introduction}

The electromagnetic novelty is that Maxwell macroscopic equations [1] are extended into a microscopic electromagnetism. The development of elementary particle physics introduced three new aspects at electromagnetic behavior. They are the electric charge be ported by particles with different flavors and spins, charge exchange $\Delta Q=0, \pm 1$ and spin working as a magnet.

Thus, given these three features, a new Lagrangian beyond Maxwell is constituted. Quantum field theory contains real processes where particles are created and destroyed. At the electric charge context this means that three types of electric charge are interwoned. They are $\triangle Q=0, \pm 1$. Consequently, given these set of three charges $\{+, 0,-\}$, it is necessary to develop an electromagnetism based on four intermediate bosons. At previous works we have developed the so-called Four Bosons Electromagnetism [2]. The next step is to introduce spin at first principles.

There is a microscopic electromagnetism based on charge exchange and spin to be understood. Maxwell's electromag- 
netism preceded the Quantum Mechanics. The electric charge was studied first than spin. However, today, there are experimental apparatus to observe on spin effects. At this work we are interested to explain the role of the spin on electric and magnetic proprieties including when unlinked to the electric charge. Although the spin concept has been known for more than 90 years, it is a current topic of investigations. Considering that spin is a space-time effect, it should be studied connected to Lorentz generators. Be defined before the EM fields by associating fields to Lorentz symmetry.

The spin was first thought of as an intrinsic angular momentum, associated with the rotation of charged particles, such as the electron. Between 1913 and 1925 the empirical phase of the spin occurred. After the publication of the two articles by Niels Bohr in July and September 1913 on which he based his atomic model[3], the Atomic Model of Bohr, there was a great semi-empirical development subsequent to the model. They were trying to reproduce, through elaborate mathematical expressions the Balmer spectral emission rays. This whole movement is faithfully reproduced in Sin-Itiro Tomonaga's book "The Story of Spin"[4], one of the founders of the current formulation of QED together with Richard Feynman and Julian Schwinger. It is very clear throughout the chapters of Tomonaga's book that there was a missing element to the former understanding of the mechanism that governs atomic spectral lines. The desperate search for mathematical expressions that reproduced the emission series was the realization that there was a concept, an idea, that was missing.

In 1921, Compton proposes the electron 'spinning'. In the quantized rotation of the electron the possible origin of the natural unit of magnetism [5]. In 1922, the experience of Stern-Gerlach [6] shows the quantization of the angular momentum of an atom. And in 1925, the concept of spin is made explicit, first by Kronig (but give up due to Pauli criticism). However, with Bohr support, Goudsmit and Uhlenbeck in order to explain the Zeeman effect, propose that besides mass and electric charge, the electron is endowed with another quantum attribute, of spatial nature, without classical analog: the spin [7]. In 1926, calculations made by Thomas confirmed the Uhlenback and Goudsmith idea of electron spin with $m_{s}= \pm \frac{1}{2}$ and magnetic moment $2 m_{s}[8]$. In 1927, Dennison discovers that proton spin is the same as electron spin. Definitely physics had to face the spin presence.

Thus, between 1925-28, the spin incorporation into the physical equations appears. With the publication of Heisenberg's Matrix Mechanics in July 1925 [9], Heisenberg and Jordan published paper introducing spin at Quantum Mechanics in 1926 [10]. After, the advent of Schrödinger's Equation in December 1926, in the famous article "An Ondulatory Theory of Atoms and Molecules", [11], Pauli, already in May 1927, launches his article where he proposes the so-called Pauli equation [12]. It couples the spin of the electron to an external magnetic field, through the so-called Pauli term of non-minimal coupling. Soon after, Pauli unsuccessfully tried to reconcile the electron spin (proposed by Goudsmit and Uhlenbeck in a non-relativistic form and used by him, Pauli, in Equation of Schrödinger, also non-relativistic) with Special Relativity. Nevertheless, it was Paul Dirac in 1928, in his two classic articles of January 2 and February 2[13], who was able to show the relativistic origin of the spin, using the so-called spinors of SL $(2, \mathrm{C})$, and writing the celebrated Dirac's Equation which is nothing more than the description of the relativistic dynamics of the spin of the electron.

Thus, in this new physical scenario of spin, the most relevant insight came from Dirac. Without even knowing in a systematic way the non-unitary representations of the finite dimension of the Lorentz Group, but knowing the relativistic spinors through the notes of Elie Cartan's course on spinors. At his famous lectures of 1913 Cartan conceived the general mathematical theory of a new category of geometric objects of a general space-time, the spinors. These classes gave rise to the book of 1938 [14]. Dirac introduced the spin of the electron in Special Relativity and retake at classical limit of his equation, the Pauli Equation of 1927. Dirac was able to show that particles having the charge and 
mass of the electron must have just the intrinsic angular momentum and magnetic moment attributed to them by Goudsmith and Uhlembeck. In this way, the concept of spin of the electron becomes well understood and, from the Dirac Equation, the relativistic corrections and the hyperfine structure of the spectral lines of the Hydrogen atom were understood more systematically.

This is a bit about spin story. It says that although the literature emphasis on the Stern-Gerlach experiment as responsible for the spin concept, its development was due to atomic physics experiments, Zeeman effect at spectroscopy [15]. Next, the magnetic momentum became an precious object of phenomenological study. First, relates quantities as electric charge, mass and spin of a particle through an dimensionless constant called gyromagnetic factor. The gyromagnetic factor become the key for the introduction of new terms like we will see next. It also reflects on elementarity. It relates that composite particles have a great gyromagnetic factor in comparation with the elementary particles. These facts make it a physical entity to be studied.

Classically, a moving particle with mass $\mathrm{m}$, velocity $\vec{v}$ and electric charge $e$, moving around a close circuit, generates a magnetic momentum dipole $\vec{\mu}$ proportional to the angular momentum $\vec{L}$. It yields

$$
\vec{\mu}=\frac{1}{2} \int d^{3} \vec{r} \rho_{e}(\vec{r} \times \vec{v})=\frac{1}{2} \frac{e}{m} \int d^{3} \vec{r} \rho_{m}(\vec{r} \times \vec{v})
$$

which gives,

$$
\vec{\mu}=g \frac{e}{2 m} \vec{L}
$$

where $g$ is introduced as the gyromagnetic factor.

Dirac equation has diverse consequences as antimatter prevision, quantum vacuum, spectral lines. However, at the scope of this work, we should note that its introduction of electron with spin- $\frac{1}{2}$ has violated the classical result at eq.(1.2) for $g=2$. Later on, when quantum field theory was established, a relationship between the gyromagnetic factor $g$ and the spin $s$ of a charged particle was obtained [16]. 
It was given by

$$
g=\frac{1}{s}
$$

Nevertheless, while eq.(1.3) supports Dirac theory for spin- $\frac{1}{2}$, it contradicts when it is applied for higher spins. At early $70^{\prime}$ Weinberg showed through $S$ matrix properties that $g \approx 2$ for massive charged particles with arbitrary spins [17]. Two decades later Ferrara, Porrati, Telegdgi have shown that any elementary (puntiform) charge particle owes at tree level the gyromagnetic factor $g=2$, independently on its spin [18]. Consequently, for spin-1, a massive charge vectorial boson $W_{\mu}$, it was necessary to introduce a non-minimal coupling given by

$$
i e F_{\mu \nu} W^{* \mu} W^{\nu}
$$

in order to obtain $g=2$.

Another consequence is on the anomalous magnetic moment. In 1947, it was observed anomalies in the factor $(g-2)$ for electron by Kusch and Foley [19]. Also the hyperfine structure hydrogen and deuterium fundamental states [20]. In response Schwinger proposed the 1-loop radioactive correction for the electron magnetic moment [21]. It became the most precise experimental result in physics. Actually one gets the following precision for the $(g-2)$ measurements: $10^{-12}$ for electron, $10^{-10}$ for muon, $10^{-2}$ for tau, $10^{-8}$ for proton, $10^{-7}$ for neutron [22].

The field evolved rapidly and many interesting developments in fundamental physics and materials science have occurred only in recent years. Derived from spin the ferromagnetism phenomena is totally related to innovation. The success story of giant magnetoresistance and its broad application to information technology - Albert Fert and Peter Grünberg, Nobel Prize for Physics in 2007 - certainly contributed to this. But it would be a fallacy to consider possible applications more important than the fundamental view provided by spintronics research. The spin is a purely quantum-mechanical entity and its interaction with the charge of electrons or the atomic environment provides a unique opportunity to understand the quantum nature of matter.

Nowadays, spintronics became an area of condensed matter physics that studies the properties of electron spin, with the goal of improving the efficiency of electronic devices and enriching them with new functionalities. Such a broad definition implies that the range of subjects falling within the umbrella of spintronics is inevitably very wide. At one extreme, researchers explore the control of unique localized spins, performed at unique atomic sites on crystals - such as nitrogen vacancy centers on diamonds - or semiconductor quantum dots. These are considered spin qubits, ideal for quantum computing in a solid-state environment. At the other extreme, researchers explore spin transport and spin dynamics in macroscale systems, coupling spin transport to spin dynamics in many ways.

The objective of this work is to study on spin effects at this Four Bosons Electromagnetism. As we know, a great achievement was obtained with spin- $\frac{1}{2}$. However, much less is known yet about spintronics-1. The literature does not report on Stern-Gerlach experiment type for spin-1. Its focus just on bosons intermediation and interaction [23]. Thus, at section 2, one studies on spin at ab initio, as consequence from Lorentz Group rotations. Spin is a fundamental physical entity that cannot depend on others. It has to be treated as a space-time intrinsec property. And so, before other physical entities be defined, we associate to the potential fields the Lorentz Group spin generator. At section 3 , field strengths and the corresponding spin-valued Lagrangian pieces are studied. These spin terms influence are notified at section 4 , where equations of motion are studied showing the fields spin interactions. Section 5 studies on 
quadruplet fields dynamics. At section 6, Noether theorem is considered. At conclusion, one understand that instead of considering as rotation, the spin must be understood as a vector. There is a vector spin $\vec{S}$ determined from the spin-valued equations.

\section{Spintronics - 1}

The scope of this work is to introduce spin from relativistic principles. Originally, spin is a quantum number derived from light invariance. However, Maxwell does not explicit such discourses. It is limited to point out to electric charge generating EM fields. However, a particle must be determinate by a chart of quantum numbers [24]. That is, spin, mass, charge, C, P, T, CPT be well defined. Nevertheless the spin is included just empirically by the coupling of the anomalous magnetic moment with the magnetic field and by the electric dipole moment with the electric field [25]. The non-relativistic Hamiltonian for EM interaction with spin- $\frac{1}{2}$ was found out to be

$$
\vec{H}=-\vec{\mu} \cdot \vec{B}-\vec{d} \cdot \vec{E}
$$

where quantically the magnetic moment is

$$
\vec{\mu}=g_{L} \frac{e \hbar}{2 m} \vec{S}
$$

Pauli equation found the term $\vec{\mu} \cdot \vec{B}$ to measure the interaction of spin with the magnetic field where $\vec{B}$ represents of the photon EM field and $\vec{S}$, the spin of matter.

The electric dipole classically is

$$
\vec{d}=\int d^{3} \vec{r} \rho(r) \vec{r}
$$

and quantically as

$$
\vec{d}=\zeta \frac{e}{2 m_{e}} \vec{S}
$$

where $\zeta$ is an dimensionless constant analogous to $g_{L}$ at magnetic case. However, quantically, these spin terms are not renormalizable.

The quest is to understand eq.(2.1) from first principles. Introduce on spin meaning more deeply. Although is not so easy to define what spin is, the phenomenological eqs(2.1-2.4) are pointing out that spin is a vectorial entity, $\vec{S}$. This is the clue to look for the spin presence. Although physics considers the spin just as a matter attribute at least we know that it appears as a vectorial object. An expression to be derived from theoretical models.

Our insight is to understand spin through Lorentz Group [26]. The insight is to couple the field with the generator of rotations, $A_{\mu, \kappa \lambda} \Sigma^{\kappa \lambda}$, in such a way that the interaction with the spin reveals the physicality of the electric dipole and magnetic mements. Given this background we are going to define the field potential as $A_{\mu ; \kappa \lambda}$ where the first index represents the space-time symmetry $x^{\prime \mu}=\Lambda_{\nu}^{\mu} x^{\nu}$ and the others are field rotation symmetry $A_{\mu}^{\prime}=\left(e^{\frac{i}{2} \omega_{\alpha \beta} \Sigma^{\alpha \beta}}\right)_{\mu}^{\nu} A_{\nu}$. And so, one obtains a spin-valued field. Something candidate to rewrite spin interactions through a renormalizable Lagrangian.

The representation $\left\{\frac{1}{2}, \frac{1}{2}\right\}$ splits that potential fields carring spin- 1 and spin- 0 . It yields, 


$$
A_{\mu} \equiv A_{\mu ; \kappa, \lambda}\left(\Sigma^{k \lambda}\right)
$$

with

$$
\left(\Sigma_{\mu \nu}\right)_{\alpha \beta}=-i\left(g_{\mu \alpha} g_{\nu \beta}-g_{\mu \beta} g_{\nu \alpha}\right)
$$

obeying the Lie Algebra

$$
\left[\Sigma_{\mu \nu}, \Sigma_{\rho_{\sigma}}\right]=g_{\mu \sigma} \Sigma_{\nu \rho}+g_{\nu \rho} \Sigma_{\mu \sigma}-g_{\mu \rho} \Sigma_{\nu \rho}-g_{\nu \sigma} \Sigma_{\mu \rho}
$$

Thus, similarly to the Yang-Mills Lie algebra valued, $A_{\mu} \equiv A_{\mu a} t_{a}$, we are going to construct a physics associated to the spin generator $\left(\Sigma_{\mu \nu}\right)_{\beta}^{\alpha}$. It considers that the fundamental information on spin comes out from Lorentz Group. Although Maxwell contains the spin present through degrees of freedom, it is under the rotation $A_{\mu}^{\prime}=\left(e^{\frac{i}{2} \omega_{\alpha \beta} \Sigma^{\alpha \beta}}\right)_{\mu}^{\nu} A_{\nu}$ that spin reveals its nature. It introduces the spin through the Lorentz group generators.

The expectation for spintronics-1 is to write the equation of motion in the following form

$$
\partial_{\nu}\left(F^{\nu \mu}+z^{[\nu \mu]}\right)=\left(F_{\alpha \beta}+z_{\alpha \beta}\right)\left(\Sigma^{\alpha \beta}\right)_{\rho}^{\mu} A^{\rho}
$$

where equation above (2.8) will work as the relativistic equation for the spin-1.

The challenge is to find out a Lagrangian providing this type of equation of motion. Although Maxwell might be derived from a spin-1 particle [27] instead of coming from charges and currents, it does not retreat the term $\vec{\mu} \cdot \vec{B}$. It does not contain the interaction with EM fields. Eqs.(2.8) performance is the coupling between the spin field and the electric and magnetic fields of the four bosons as (2.1). Its proposal is to couple spin with the granular and collective fields strengths tensors, like the terms $\vec{B} \cdot \vec{S}, \vec{E} \cdot \vec{S}, \vec{e} \times \vec{S}$ and so on. At this way, from (2.5) as origin one expects to write first a Lagrangian with explicit terms of spin, and then, obtain the desired equation of motion (2.8). There is a hidden vector $\vec{S}$ to be found.

\section{Spin-Valued Four Bosons Lagrangian}

The model Four Bosons EM introduces a quadruplet as intermediating the electric charge exchange. Originally, its physicality expresses the charge exchange $\Delta Q=0, \pm 1$ through four potential fields $A_{\mu I}=\left\{A_{\mu}, U_{\mu}, V_{\mu}^{ \pm}\right\}$where $A_{\mu}$ means the usual photon, $U_{\mu}$ massive photon, $V_{\mu}^{ \pm}$massive charge photons transforming under the abelian $U(1) \times S O(2)_{\text {global }}$ symmetry.

The new fact is to associate the spin to these potential fields. Bosonic spin-1 particles were, in general, discovered after the fermionic spin $\frac{1}{2}$ particles, and incorporated as fundamental in characterizing physical interactions. However, EM is being the theory for electric charge and spin. And so, a spin step forward to the four bosons electromagnetism is expected. Keeping eq.(2.5) in mind, one gets the gauge transformations 


$$
\begin{aligned}
& A_{\mu ; \kappa \lambda}^{\prime}\left(\Sigma^{\kappa \lambda}\right)=A_{\mu ; \kappa \lambda}\left(\Sigma^{\kappa \lambda}\right)+k_{1} \partial_{\mu} \alpha \\
& U_{\mu ; \kappa \lambda}^{\prime}\left(\Sigma^{\kappa \lambda}\right)=U_{\mu ; \kappa \lambda}\left(\Sigma^{\kappa \lambda}\right)+k_{2} \partial_{\mu} \alpha \\
& V_{\mu ; \kappa \lambda}^{\prime+}\left(\Sigma^{\kappa \lambda}\right)=e^{i q \alpha} V_{\mu ; \kappa \lambda}^{+}\left(\Sigma^{\kappa \lambda}\right)+k_{+} \partial_{\mu} \alpha \\
& V_{\mu ; \kappa \lambda}^{\prime-}\left(\Sigma^{\kappa \lambda}\right)=e^{-i q \alpha} V_{\mu ; \kappa \lambda}^{-}\left(\Sigma^{\kappa \lambda}\right)+k_{-} \partial_{\mu} \alpha
\end{aligned}
$$

Thus, in order to look for the equation of motion (2.8), we are going to study a spintronic gauge theory based on eq.(3.1) symmetry. Consider under eq.(2.5) the respective granular and collective fields strengths and mass obtained previously with the Four Bosons Electromagnetic Model [2]. Develop from the initial Four Fields Lagrangian the Spin-Valued Four Bosons Lagrangian.

A new Lagrangian able to build the so-called spintronics of spin-1 will be studied. Integrate eqs.(2.5) and (3.1). The mapping between the former and the new Lagrangian is given as.

$$
\begin{aligned}
& F_{\mu \nu} \rightarrow\left(\Sigma_{\mu \nu}\right)_{\rho_{\sigma}} F^{\rho_{\sigma}} \\
& z_{[\mu \nu]} \rightarrow\left(\Sigma_{\mu \nu}\right)_{\rho_{\sigma}} z^{\rho_{\sigma}} \\
& \beta_{I} S_{\mu \nu}^{I} \rightarrow\left(\Sigma_{\mu \nu}\right)_{\rho_{\sigma}} \beta_{I} S^{I \rho_{\sigma}}
\end{aligned}
$$

and so on.

We are going to rewrite every Four Bosons EM Lagrangian sector separately. Considering first the kinetic sector, one originally gets

$$
\mathcal{L}=\mathcal{L}_{K}+\mathcal{L}_{G F}+\mathcal{L}_{I}
$$

where the kinect term,

$$
\mathcal{L}_{K}=\mathcal{L}_{K}^{A}+\mathcal{L}_{K}^{S}
$$

is given by

$$
\begin{aligned}
\mathcal{L}_{K}^{A} & =a_{1} F_{\mu \nu} F^{\mu \nu}+a_{2} U_{\mu \nu} U^{\mu \nu}+2 a_{3} V_{\mu \nu}^{+} V^{\mu \nu-}, \\
\mathcal{L}_{K}^{S} & =b_{(11)} S_{\mu \nu}^{1} S^{\mu \nu 1}+b_{(22)} S_{\mu \nu}^{2} S^{\mu \nu 2} \\
& +2 b_{(33)} S_{\mu \nu}^{+} S^{\mu \nu-}+c_{(11)} S_{\mu}^{\mu 1} S_{\nu}^{\nu 1}+c_{(22)} S_{\mu}^{\mu 2} S_{\nu}^{\nu 2} \\
& +2 c_{(12)} S_{\mu}^{\mu 1} S_{\nu}^{\nu 2}+2 c_{(33)} S_{\mu}^{\mu+} S_{\nu}^{\nu-},
\end{aligned}
$$

with the following definitions for the granular field strength tensors

$$
\begin{array}{lll}
F_{\mu \nu} \equiv \partial_{\mu} A_{\nu}-\partial_{\nu} A_{\mu}, & U_{\mu \nu} \equiv \partial_{\mu} U_{\nu}-\partial_{\nu} U_{\mu}, & V_{\mu \nu}^{ \pm} \equiv \partial_{\mu} V_{\nu}^{ \pm}-\partial_{\nu} V_{\mu}^{ \pm}, \\
S_{\mu \nu}^{1} \equiv \partial_{\mu} A_{\nu}+\partial_{\nu} A_{\mu}, & S_{\mu \nu}^{2} \equiv \partial_{\mu} U_{\nu}+\partial_{\nu} U_{\mu}, & S_{\mu \nu}^{ \pm} \equiv \partial_{\mu} V_{\nu}^{ \pm}+\partial_{\nu} V_{\mu}^{ \pm} .
\end{array}
$$

For the gauge-fixing term, 


$$
\mathcal{L}_{G F}=\frac{1}{4} \xi_{(11)} S_{\mu}^{\mu 1} S_{\nu}^{\nu 1}+\frac{1}{4} \xi_{(22)} S_{\mu}^{\mu 2} S_{\nu}^{\nu 2}+\frac{1}{2} \xi_{(12)} S_{\mu}^{\mu 1} S_{\nu}^{\nu 2}+\frac{1}{2} \xi_{(33)} S_{\mu}^{\mu+} S_{\nu}^{\nu-}
$$

For the mass term,

$$
\mathcal{L}_{m}=-\frac{1}{2} \mu_{U}^{2} U_{\mu} U^{\mu}-\mu_{V}^{2} V_{\mu}^{+} V^{\mu-}
$$

Notice that eq. (3.9) does not require the spontaneous symmetry breaking. The mass inclusion is given by the symmetry of differences. The gauge invariance for the term $\mathcal{L}_{m}$ is obtained as a whole.

For the interaction part, where the collective fields are defined at Appendix A, one gets

$$
\mathcal{L}_{I}=\mathcal{L}_{3}+\mathcal{L}_{4}
$$

where

$$
\mathcal{L}_{3}=\mathcal{L}_{3}^{A}+\mathcal{L}_{3}^{S}+\mathcal{L}_{3}^{s t}
$$

with

$$
\begin{aligned}
& \mathcal{L}_{3}^{A}=4 b_{1} F_{\mu \nu} \stackrel{[12]}{z}[\mu \nu]+4 b_{2} U_{\mu \nu} \stackrel{[12]}{z}[\mu \nu]+4 b_{1} F_{\mu \nu}{ }^{[+--]}[\mu \nu]+4 b_{2} U_{\mu \nu}{ }^{[+-}{ }^{[}[\mu \nu]+4 \beta_{1} F_{\mu \nu}{ }^{(+-)}{ }^{[\mu \nu]}+
\end{aligned}
$$

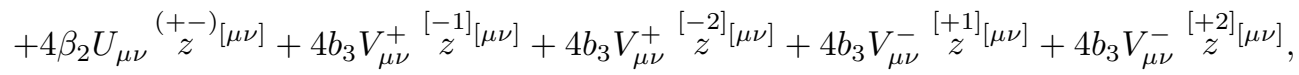

$$
\begin{aligned}
& \mathcal{L}_{3}^{S}=2 \beta_{1} S_{\mu \nu}^{1} \stackrel{(11)}{z} \mu \nu+4 \beta_{1} S_{\mu \nu}^{1} \stackrel{(12)}{z} \mu \nu+2 \beta_{1} S_{\mu \nu}^{1} \stackrel{(22)}{z} \mu \nu+4 \beta_{1} S_{\mu \nu}^{1}{ }^{+} \bar{z}^{3} \mu \nu+ \\
& +2 \beta_{2} S_{\mu \nu}^{2} \stackrel{(11)}{z} \mu \nu+4 \beta_{2} S_{\mu \nu}^{2} \stackrel{(12)}{z} \mu \nu+2 \beta_{2} S_{\mu \nu}^{2} \stackrel{(22)}{z} \mu \nu+4 \beta_{2} S_{\mu \nu}^{2}{ }^{+3}{ }^{3} \mu \nu+ \\
& +4 \beta_{3} S_{\mu \nu}^{+} \stackrel{(-1)}{z} \mu \nu+4 \beta_{3} S_{\mu \nu}^{+} \stackrel{(-2)}{z} \mu \nu+4 \beta_{3} S_{\mu \nu}^{-} \stackrel{(+1)}{z} \mu \nu+4 \beta_{3} S_{\mu \nu}^{-} \stackrel{(+2)}{z} \mu \nu+ \\
& +2 \rho_{1} S_{\mu}^{\mu 1} \stackrel{(11)}{z}{ }_{\nu}+4 \rho_{1} S_{\mu}^{\mu 1} \stackrel{(12)}{z}{ }_{\nu}+2 \rho_{1} S_{\mu}^{\mu 1}{\stackrel{(22)}{z}{ }_{\nu}}^{(2)}+4 \rho_{1} S_{\mu}^{\mu 1}+\bar{z}_{\nu}^{-3}+ \\
& +2\left(\beta_{1}+4 \rho_{1}\right) S_{\mu}^{\mu 1} \stackrel{(11)}{\omega}{ }_{\nu}+4\left(\beta_{1}+4 \rho_{1}\right) S_{\mu}^{\mu 1} \stackrel{(12)}{\omega}_{\nu}+2\left(\beta_{1}+4 \rho_{1}\right) S_{\mu}^{\mu 1} \stackrel{(22)}{\omega}_{\nu}+ \\
& +4\left(\beta_{1}+4 \rho_{1}\right) S_{\mu}^{\mu 1}{ }^{+}-{ }^{3}{ }_{\nu}+2 \rho_{2} S_{\mu}^{\mu 2} \stackrel{(11)}{z}_{\nu}+4 \rho_{2} S_{\mu}^{\mu 2} \stackrel{(12)}{z}_{\nu}+2 \rho_{2} S_{\mu}^{\mu 2} \stackrel{(22)}{z} \nu_{\nu}+ \\
& +4 \rho_{2} S_{\mu}^{\mu 2}{ }_{z}^{+3}{ }_{\nu}++2\left(\beta_{2}+4 \rho_{2}\right) S_{\mu}^{\mu 2} \stackrel{(11)}{\omega}{ }_{\nu}+4\left(\beta_{2}+4 \rho_{2}\right) S_{\mu}^{\mu 2} \stackrel{(12)}{\omega}_{\nu}+ \\
& +2\left(\beta_{2}+4 \rho_{2}\right) S_{\mu}^{\mu 2} \stackrel{(22)}{\omega}_{\nu}+4\left(\beta_{2}+4 \rho_{2}\right) S_{\mu}^{\mu 2}{ }^{+-3}{ }_{\nu}+4\left(\beta_{3}+4 \rho_{3}\right) S_{\mu}^{\mu+}{\stackrel{(-1)}{\omega}{ }_{\nu}}+ \\
& +4\left(\beta_{3}+4 \rho_{3}\right) S_{\mu}^{\mu+} \stackrel{(-2)}{\omega}_{\nu}++4 \rho_{3} S_{\mu}^{\mu+}{ }_{z}^{(-1)}{ }_{\nu}+4 \rho_{3} S_{\mu}^{\mu+}{ }_{z}^{(-2)}{ }_{\nu}+4 \rho_{3} S_{\mu}^{\mu-}{ }_{z}^{(+2)}{ }_{\nu}+ \\
& +4\left(\beta_{3}+4 \rho_{3}\right) S_{\mu}^{\mu-} \stackrel{(+1)}{\omega}_{\nu}+4\left(\beta_{3}+4 \rho_{3}\right) S_{\mu}^{\mu-} \stackrel{(+2)}{\omega}_{\nu}+4 \rho_{3} S_{\mu}^{\mu-}{ }_{z}^{(+1)} \nu_{\nu}
\end{aligned}
$$


and

$$
\begin{aligned}
\mathcal{L}_{3}^{s t} & =\epsilon^{\mu \nu \rho \sigma}\left\{2\left(\alpha_{0} A_{\mu}+\alpha_{1} U_{\mu}\right)\left[i\left(V_{\nu}^{+} V_{\rho \sigma}^{-}-V_{\nu}^{-} V_{\rho \sigma}^{+}\right)\right]+\left(\alpha_{0} A_{\rho \sigma}+\alpha_{1} U_{\rho \sigma}\right)\left[i\left(\stackrel{[+-}{z}_{\mu \nu}+\stackrel{[-+]}{z}_{\mu \nu}\right)\right]\right. \\
& \left.+4 \stackrel{[12]}{z}_{\mu \nu}\left(\alpha_{0} A_{\rho \sigma}+\alpha_{1} U_{\rho \sigma}\right)\right\} .
\end{aligned}
$$

For the quadrilinear term

$$
\mathcal{L}_{4}=\mathcal{L}_{4}^{A}+\mathcal{L}_{4}^{S}+\mathcal{L}_{4}^{s t}
$$

where

$$
\begin{aligned}
& \mathcal{L}_{4}^{A}=2 \stackrel{[12]}{z}_{[\mu \nu]}^{[12]}{ }^{[\mu \nu]}+2 \stackrel{[12]}{z}_{[\mu \nu]}^{[21]}{ }^{[\mu \nu]}+4 \stackrel{[13+]}{z}_{[\mu \nu]}^{[13-]}{ }^{[\mu \nu]}+
\end{aligned}
$$

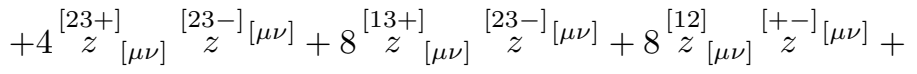

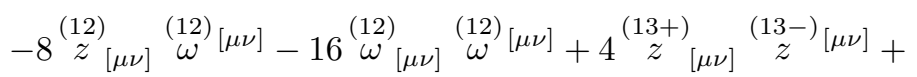

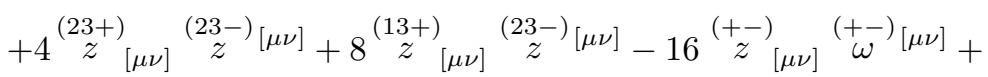

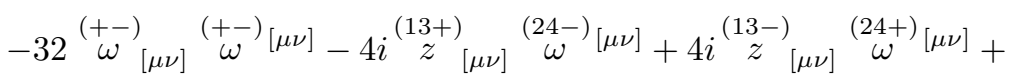

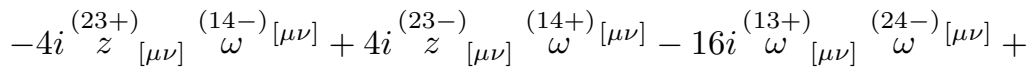

$$
\begin{aligned}
& +16 i \stackrel{(13-)}{\omega}_{[\mu \nu]}^{(24+)} \stackrel{(\omega \nu]}{\omega}-2 i \stackrel{(14+)}{z}_{[\mu \nu]}^{(23-)}{ }_{z}^{[\mu \nu]}+2 i i_{z}^{(14-)}{ }_{[\mu \nu]}^{(23+)}{ }_{z}^{[\mu \nu]}+ \\
& +16 i \stackrel{(14+)}{\omega}_{[\mu \nu]}^{(23-)}\left[{ }^{[\mu \nu]}-16 i i^{(14-)}{ }_{[\mu \nu]}^{(23+)}{ }^{(\mu \nu]}+2 i{ }_{z}^{(13+)}{ }_{[\mu \nu]}^{(24-)}{ }_{z}^{[\mu \nu]}+\right.
\end{aligned}
$$

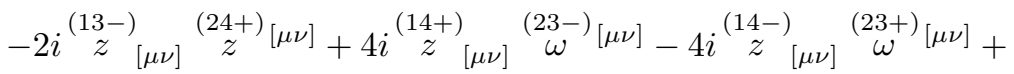

$$
\begin{aligned}
& +4 i \stackrel{(24+)}{z}_{[\mu \nu]}^{(13-)} \stackrel{(\mu \nu]}{\omega}^{\left[\mu i^{(24-)}\right.}{ }_{[\mu \nu]}^{(13+)} \stackrel{(\mu \nu]}{ }^{(\mu \nu]} \text {, }
\end{aligned}
$$




$$
\begin{aligned}
& \mathcal{L}_{4}^{S}=2 \stackrel{[12]}{z}_{(\mu \nu)} \stackrel{[12]}{z}(\mu \nu)^{[12} \stackrel{[12]}{z}(\mu \nu)^{[21]}(\mu \nu)+4 \stackrel{[13+]}{z}(\mu \nu)_{z}^{[13-]}(\mu \nu)+
\end{aligned}
$$

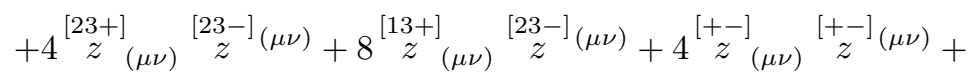

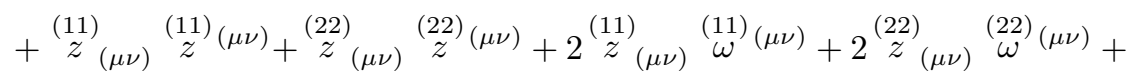

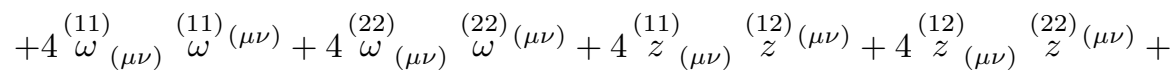

$$
\begin{aligned}
& +8 \stackrel{(12)}{z}_{(\mu \nu)} \stackrel{(11)}{\omega}^{(\mu \nu)}+8 \stackrel{(12)}{z}_{(\mu \nu)} \stackrel{(22)}{\omega}^{(\mu \nu)}+16 \stackrel{(11)}{\omega}_{(\mu \nu)} \stackrel{(12)}{\omega}(\mu \nu)^{(12)}+16 \stackrel{(12)}{\omega}_{(\mu \nu)} \stackrel{(22)}{\omega}^{(\mu \nu)}+ \\
& +2 \stackrel{(11)}{z}_{(\mu \nu)} \stackrel{(22)}{z}(\mu \nu)^{(121)}\left(\stackrel{(11)}{z}_{(\mu \nu)}+\stackrel{(22)}{z}_{(\mu \nu)}\right){ }^{+-3}(\mu \nu)+4 \stackrel{(12)}{z}_{(\mu \nu)} \stackrel{(12)}{z}(\mu \nu)^{(12)}
\end{aligned}
$$

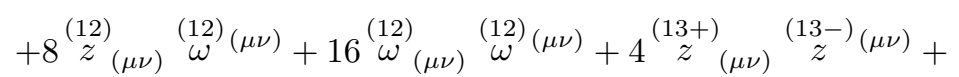

$$
\begin{aligned}
& +4 \stackrel{(23+)}{z}_{(\mu \nu)}^{(23-)} z^{(\mu \nu)}+8 \stackrel{(13+)}{z}_{(\mu \nu)}^{(23-)}{ }^{(\mu \nu)}+8 \stackrel{(12)}{z}_{(\mu \nu)}+\bar{z}^{-3}(\mu \nu)+ \\
& +4{ }^{+-3}{ }_{(\mu \nu)}^{+-4}{ }^{4}(\mu \nu)+4 i{ }_{z}^{(13+)}(\mu \nu) \stackrel{(24-)}{\omega}(\mu \nu)-4 i i_{(\mu \nu)}^{(13-)} \stackrel{(24+)}{\omega}(\mu \nu)+
\end{aligned}
$$

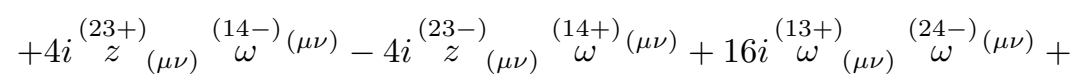

$$
\begin{aligned}
& -16 i \stackrel{(13-)}{\omega}_{(\mu \nu)}^{(24+)} \stackrel{(\mu \nu)}{\omega}^{\left(\mu i^{(14+)}\right.}{ }_{(\mu \nu)}^{(23-)}{ }^{(\mu \nu)}-2 i \stackrel{(14-)}{z}(\mu \nu)_{z}^{(23+)}(\mu \nu)+ \\
& -16 i \stackrel{(14+)}{\omega}_{(\mu \nu)}^{(23-)} \stackrel{(\mu \nu)}{\omega}^{(23}+16 i \stackrel{(14-)}{\omega}_{(\mu \nu)}^{(23+)} \stackrel{(\mu \nu)}{\omega}^{(13+)} i_{(\mu \nu)}^{(24-)}{ }_{z}^{(\mu \nu)}+
\end{aligned}
$$

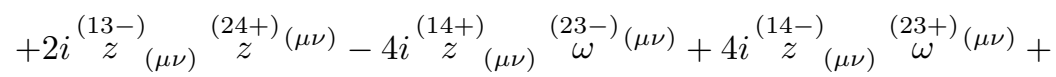

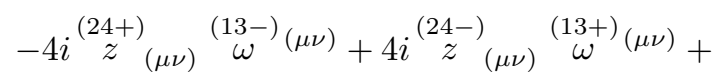

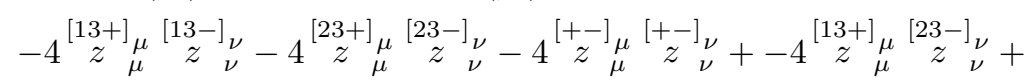

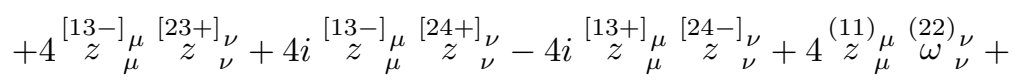

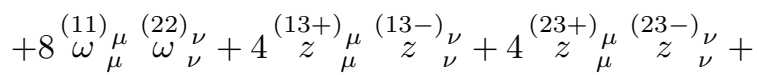

$$
\begin{aligned}
& +16 \stackrel{(13+)}{z}_{\mu}^{(13-)} \omega_{\nu}+16 \stackrel{(23+)}{z}{ }_{\mu} \stackrel{(23-)}{\omega}_{\nu}+32 \stackrel{(13+)}{\omega}_{\mu}^{(13-)}{ }_{\nu}{ }_{\nu}+
\end{aligned}
$$

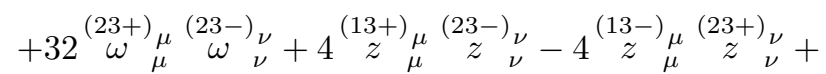

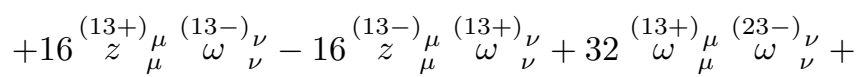

$$
\begin{aligned}
& -32 \stackrel{(13-)}{\omega}_{\mu}^{(23+)} \stackrel{\omega}{\omega}_{\nu}-4{ }_{z}^{(+-)}{ }_{\mu}\left(+z_{\nu}^{(+)}{ }_{\nu}+8{ }^{+-3}{ }_{\mu}+\bar{\omega}_{\nu}{ }_{\nu}+16{ }^{+-3}{ }_{\mu}+\bar{\omega}_{\nu}{ }_{\nu}+\right. \\
& +8\left\{\stackrel{(11)}{z}_{\mu}+\stackrel{(22)}{z}_{\mu}+2 \stackrel{(11)}{\omega}_{\mu}^{\mu}+2 \stackrel{(22)}{\omega}_{\mu}^{\mu}+2 \stackrel{(12)}{z}_{\mu}+4 \stackrel{(12)}{\omega}_{\mu}^{\mu}\right\}{ }^{+-3}{ }_{\nu}
\end{aligned}
$$

$$
\mathcal{L}_{4}^{s t}=\epsilon^{\mu \nu \rho \sigma}\left\{4 \stackrel{[12]}{z}_{\mu \nu}^{[12]} \stackrel{[}{\rho \sigma}_{\rho \sigma}+4 \stackrel{[12]}{z}_{\mu \nu}\left(\stackrel{[+-}{z}_{\rho \sigma}+\stackrel{[-+]}{z}_{\rho \sigma}\right)+\left(\stackrel{[+-]}{z}_{\mu \nu}+\stackrel{[-+]}{z}_{\mu \nu}\right)\left({ }^{[+-]} z_{\rho \sigma}+\stackrel{[-+]}{z} \rho \sigma_{\rho \sigma}\right)\right\}
$$

where the collective fields $z_{\mu \nu}$ are defined at Appendix A.

Thus, the objective of this work is to construct a spin physics associated to the spin generator $\left(\Sigma_{\mu \nu}\right)_{\beta}^{\alpha}$. This means to rewrite the Lagrangian at eq.(3.3) as 


$$
\mathcal{L}^{\text {spin }}=\mathcal{L}_{K}^{\text {spin }}+\mathcal{L}_{G F}^{\text {spin }}+\mathcal{L}_{I}^{\text {spin }}
$$

From eqs.(2.5-2.6), we get the following relationships

$$
\begin{aligned}
& \left(\Sigma^{\mu \nu}\right)_{\sigma}^{\rho}\left(\Sigma^{\kappa \lambda}\right)_{\rho}^{\sigma} F_{\mu \nu} F_{\kappa \lambda}=4 F_{\mu \nu} F^{\mu \nu} \\
& \left(\Sigma^{\mu \kappa}\right)_{\sigma}^{\rho}\left(\Sigma^{\nu \lambda}\right)_{\rho}^{\sigma} F_{\mu \nu} F_{\kappa \lambda}=2 F_{\mu \nu} F^{\mu \nu}
\end{aligned}
$$

So we can write,

$$
F_{\mu \nu} F^{\mu \nu}=\frac{1}{8}\left(\Sigma^{\mu \nu}\right)_{\sigma}^{\rho}\left(\Sigma^{\kappa \lambda}\right)_{\rho}^{\sigma} F_{\mu \nu} F_{\kappa \lambda}+\frac{1}{4}\left(\Sigma^{\mu \kappa}\right)_{\sigma}^{\rho}\left(\Sigma^{\nu \lambda}\right)_{\rho}^{\sigma} F_{\mu \nu} F_{\kappa \lambda}
$$

The new $\mathcal{L}_{K}^{\text {spin A }}$ becomes,

$$
\begin{aligned}
& \mathcal{L}_{K}^{\operatorname{spin} A}=\frac{1}{8}\left(\Sigma^{\mu \nu}\right)_{\sigma}^{\rho}\left(\Sigma^{\kappa \lambda}\right)_{\rho}^{\sigma} a_{1} F_{\mu \nu} F_{\kappa \lambda}+\frac{1}{4}\left(\Sigma^{\mu \kappa}\right)_{\sigma}^{\rho}\left(\Sigma^{\nu \lambda}\right)_{\rho}^{\sigma} a_{1} F_{\mu \nu} F_{\kappa \lambda}+ \\
& +\frac{1}{8}\left(\Sigma^{\mu \nu}\right)_{\sigma}^{\rho}\left(\Sigma^{\kappa \lambda}\right)_{\rho}^{\sigma} a_{2} U_{\mu \nu} U_{\kappa \lambda}+\frac{1}{4}\left(\Sigma^{\mu \kappa}\right)_{\sigma}^{\rho}\left(\Sigma^{\nu \lambda}\right)_{\rho}^{\sigma} a_{2} U_{\mu \nu} U_{\kappa \lambda}+ \\
& +\frac{1}{4}\left(\Sigma^{\mu \nu}\right)_{\sigma}^{\rho}\left(\Sigma^{\kappa \lambda}\right)_{\rho}^{\sigma} a_{3} V_{\mu \nu}^{+} V_{\kappa \lambda}^{-}+\frac{1}{2}\left(\Sigma^{\mu \kappa}\right)_{\sigma}^{\rho}\left(\Sigma^{\nu \lambda}\right)_{\rho}^{\sigma} a_{3} V_{\mu \nu}^{+} V_{\kappa \lambda}^{-}
\end{aligned}
$$

Applying eq.(2.6), we get

$$
\mathcal{L}_{K}^{\operatorname{spin} A}=a_{1} F_{\mu \nu} F^{\mu \nu}+a_{2} U_{\mu \nu} U^{\mu \nu}+2 a_{3} V_{\mu \nu}^{+} V^{-\mu \nu}
$$

Comparing this sector $\mathcal{L}_{K}^{\text {spin } A}$ with the term $\mathcal{L}_{K}^{A}$ from the original four bosons Lagrangian

$$
\mathcal{L}_{K}^{\operatorname{spin} A}=\mathcal{L}_{K}^{A}+\Delta_{K}^{\mathrm{spin}, A}
$$

where $\Delta_{K}^{\mathrm{spin}, A}=0$ means that there is no spin contribution being introduced in the anti-symmetric kinetic granular sector.

For the symmetric kinetic sector, we can couple the Lorentz generator term with the granular terms. It gives,

$$
\begin{aligned}
& \left(\Sigma^{\mu \nu}\right)_{\sigma}^{\rho}\left(\Sigma^{\kappa \lambda}\right)_{\rho}^{\sigma} S_{\mu \nu} S_{\kappa \lambda}=0 \\
& \left(\Sigma^{\mu \kappa}\right)_{\sigma}^{\rho}\left(\Sigma^{\nu \lambda}\right)_{\rho}^{\sigma} S_{\mu \nu} S_{\kappa \lambda}=-2 S_{\mu \nu} S^{\mu \nu}+2 \mathbf{s} S_{\mu}^{\mu} S_{\nu}^{\nu} \\
& \left(\Sigma_{\mu \nu}\right)_{\sigma}^{\rho}\left(\Sigma^{\mu \nu}\right)_{\rho}^{\sigma} S_{\alpha}^{\alpha} S_{\beta}^{\beta}=-24 S_{\mu}^{\mu} S_{\nu}^{\nu}
\end{aligned}
$$


So we can obtain a new Lagrangian $\mathcal{L}_{K}^{\text {spin } S}$

$$
\begin{aligned}
& \mathcal{L}_{K}^{\operatorname{spin} S}=-\frac{1}{2}\left(\Sigma^{\mu \kappa}\right)_{\sigma}^{\rho}\left(\Sigma^{\nu \lambda}\right)_{\rho}^{\sigma} b_{(11)} S_{\mu \nu}^{1} S_{\kappa \lambda}^{1}-\frac{1}{2}\left(\Sigma^{\mu \kappa}\right)_{\sigma}^{\rho}\left(\Sigma^{\nu \lambda}\right)_{\rho}^{\sigma} b_{(22)} S_{\mu \nu}^{2} S_{\kappa \lambda}^{2}+ \\
& -\frac{1}{4}\left(\Sigma^{\mu \kappa}\right)_{\sigma}^{\rho}\left(\Sigma^{\nu \lambda}\right)_{\rho}^{\sigma} b_{(33)} S_{\mu \nu}^{+} S_{\kappa \lambda}^{-}-\frac{1}{24}\left(\Sigma_{\kappa \lambda}\right)_{\sigma}^{\rho}\left(\Sigma^{\kappa \lambda}\right)_{\rho}^{\sigma} c_{(11)} S_{\alpha}^{\alpha 1} S_{\beta}^{\beta 1}+ \\
& -\frac{1}{24}\left(\Sigma_{\kappa \lambda}\right)_{\sigma}^{\rho}\left(\Sigma^{\kappa \lambda}\right)_{\rho}^{\sigma} c_{(22)} S_{\alpha}^{\alpha 2} S_{\beta}^{\beta 2}-\frac{1}{12}\left(\Sigma_{\kappa \lambda}\right)_{\sigma}^{\rho}\left(\Sigma^{\kappa \lambda}\right)_{\rho}^{\sigma} c_{(12)} S_{\alpha}^{\alpha 1} S_{\beta}^{\beta 2}+ \\
& -\frac{1}{12}\left(\Sigma_{\kappa \lambda}\right)_{\sigma}^{\rho}\left(\Sigma^{\kappa \lambda}\right)_{\rho}^{\sigma} c_{(33)} S_{\alpha}^{\alpha+} S_{\beta}^{\beta-}
\end{aligned}
$$

Using the relations in the previous section, we have

$$
\begin{aligned}
& \mathcal{L}_{K}^{\operatorname{spin} S}=b_{(11)} S_{\mu \nu}^{1} S^{1 \mu \nu}+b_{(22)} S_{\mu \nu}^{2} S^{2 \mu \nu}+b_{(33)} S_{\mu \nu}^{+} S^{-\mu \nu}+ \\
& +\left(c_{(11)}-b_{(11)}\right) S_{\alpha}^{1 \alpha} S_{\beta}^{1 \beta}+c_{(12)} S_{\alpha}^{1 \alpha} S_{\beta}^{2 \beta}+ \\
& +\left(c_{(22)}-b_{(22)}\right) S_{\alpha}^{2 \alpha} S_{\beta}^{2 \beta}+\left(c_{(33)}-b_{(33)}\right) S_{\alpha}^{1 \alpha} S_{\beta}^{1 \beta}
\end{aligned}
$$

which yields,

$$
\begin{aligned}
& \mathcal{L}_{K}^{\operatorname{spin} S}=\mathcal{L}_{K}^{S}+\Delta_{K}^{\operatorname{spin}, S} \\
& \Delta_{K}^{\operatorname{spin}, S}=-b_{(11)} S_{\alpha}^{1 \alpha} S_{\beta}^{1 \beta}-b_{(22)} S_{\alpha}^{2 \alpha} S_{\beta}^{2 \beta}-b_{(33)} S_{\alpha}^{+\alpha} S_{\beta}^{-\beta}
\end{aligned}
$$

Eq. (3.28) shows that there is a spin-valued contribution at longitudinal sector.

For the gauge fixing term,

$$
\begin{aligned}
& \mathcal{L}_{G F}^{\mathrm{spin}}=-\frac{1}{96}\left(\Sigma_{\kappa \lambda}\right)_{\sigma}^{\rho}\left(\Sigma^{\kappa \lambda}\right)_{\rho}^{\sigma} \xi_{(11)} S_{\alpha}^{1 \alpha} S_{\beta}^{1 \beta}-\frac{1}{96}\left(\Sigma_{\kappa \lambda}\right)_{\sigma}^{\rho}\left(\Sigma^{\kappa \lambda}\right)_{\rho}^{\sigma} \xi_{(22)} S_{\alpha}^{2 \alpha} S_{\beta}^{2 \beta}+ \\
& -\frac{1}{48}\left(\Sigma_{\kappa \lambda}\right)_{\sigma}^{\rho}\left(\Sigma^{\kappa \lambda}\right)_{\rho}^{\sigma} \xi_{(12)} S_{\alpha}^{1 \alpha} S_{\beta}^{2 \beta}-\frac{1}{48}\left(\Sigma_{\kappa \lambda}\right)_{\sigma}^{\rho}\left(\Sigma^{\kappa \lambda}\right)_{\rho}^{\sigma} \xi_{(33)} S_{\alpha}^{+\alpha} S_{\beta}^{-\beta},
\end{aligned}
$$

So, simplifying the above expression, one gets

$$
\mathcal{L}_{G F}^{\mathrm{spin}}=\frac{1}{4} \xi_{(11)} S_{\alpha}^{1 \alpha} S_{\beta}^{1 \beta}+\frac{1}{4} \xi_{(22)} S_{\alpha}^{2 \alpha} S_{\beta}^{2 \beta}+\frac{1}{2} \xi_{(12)} S_{\alpha}^{2 \alpha} S_{\beta}^{2 \beta}+\frac{1}{2} \xi_{(33)} S_{\alpha}^{+\alpha} S_{\beta}^{-\beta}
$$

and,

$$
\begin{aligned}
& \mathcal{L}_{G F}^{\text {spin }}=\mathcal{L}_{G F}+\Delta_{G F}^{\text {spin }} \\
& \Delta_{G F}^{\text {spin }}=0
\end{aligned}
$$

For the mass term, we can use the following expressions 


$$
\begin{aligned}
& \left(\Sigma_{\mu \nu}\right)_{\sigma}^{\rho}\left(\Sigma^{\mu \nu}\right)_{\rho}^{\sigma} U_{\alpha} U^{\alpha}=-24 U_{\mu} U^{\mu} \\
& \left(\Sigma_{\alpha \beta}\right)_{\sigma}^{\rho}\left(\Sigma^{\beta}{ }_{\kappa}\right)_{\rho}^{\sigma} U^{\alpha} U^{\kappa}=-6 U_{\mu} U^{\mu}
\end{aligned}
$$

to write

$$
U_{\mu} U^{\mu}=-\frac{1}{48}\left(\Sigma_{\mu \nu}\right)_{\sigma}^{\rho}\left(\Sigma^{\mu \nu}\right)_{\rho}^{\sigma} U_{\alpha} U^{\alpha}-\frac{1}{12}\left(\Sigma^{\alpha \beta}\right)_{\sigma}^{\rho}\left(\Sigma_{\beta}{ }^{\kappa}\right)_{\rho}^{\sigma} U_{\alpha} U_{\kappa}
$$

Then, we can write

$$
\begin{aligned}
& \mathcal{L}_{M}^{\text {spin }}=\frac{1}{48}\left(\Sigma_{\kappa \lambda}\right)_{\sigma}^{\rho}\left(\Sigma^{\kappa \lambda}\right)_{\rho}^{\sigma} \mu_{U}^{2} U_{\alpha} U^{\alpha}+\frac{1}{12}\left(\Sigma^{\alpha \lambda}\right)_{\sigma}^{\rho}\left(\Sigma_{\lambda}^{\beta}{ }_{\lambda}\right)_{\rho}^{\sigma} \mu_{U}^{2} U_{\alpha} U_{\beta}+ \\
& +\frac{1}{24}\left(\Sigma_{\kappa \lambda}\right)_{\sigma}^{\rho}\left(\Sigma^{\kappa \lambda}\right)_{\rho}^{\sigma} \mu_{ \pm}^{2} V_{\alpha}^{+} V^{-\alpha}+\frac{1}{6}\left(\Sigma^{\alpha \lambda}\right)_{\sigma}^{\rho}\left(\Sigma_{\lambda}^{\beta}{ }_{\rho}^{\sigma} \mu_{ \pm}^{\sigma} \mu_{ \pm}^{2} V_{\alpha}^{+} V_{\beta}^{-}\right.
\end{aligned}
$$


Using the expressions derived in the previous section, one gets

$$
\mathcal{L}_{m}=-\frac{1}{2} \mu_{U}^{2} U_{\sigma} U^{\sigma}-\mu_{ \pm}^{2} V_{\alpha}^{+} V_{\beta}^{-}
$$

Then,

$$
\begin{aligned}
& \mathcal{L}_{m}^{\text {spin }}=\mathcal{L}_{m}+\Delta_{m}^{\text {spin }} \\
& \Delta_{m}^{\text {spin }}=0
\end{aligned}
$$

Next step is to study the spin influence on interaction sector, at eq.(3.10). Initially, consider the trilinear antisymmetric term

$$
\begin{aligned}
& \mathcal{L}_{3}^{\text {spin } A}=\left(\frac{1}{4}\left(\Sigma^{\mu \nu}\right)_{\sigma}^{\rho}\left(\Sigma^{\kappa \lambda}\right)_{\rho}^{\sigma}+\frac{1}{2}\left(\Sigma^{\mu \kappa}\right)_{\sigma}^{\rho}\left(\Sigma^{\nu \lambda}\right)_{\rho}^{\sigma}\right)\left(4 b_{1} F_{\mu \nu} \stackrel{[12]}{z}_{[\kappa \lambda]}+4 b_{2} U_{\mu \nu}{ }_{[\kappa \lambda]}^{[12]}+\right. \\
& +4 b_{1} F_{\mu \nu} \stackrel{[+-]}{z}[\kappa \lambda]_{[\kappa \lambda}+4 b_{2} U_{\mu \nu} \stackrel{[+-]}{z}[\kappa \lambda]_{[\kappa \lambda]}+4 \beta_{1} F_{\mu \nu} \stackrel{(+-)}{z}_{[\kappa \lambda]}+4 \beta_{2} U_{\mu \nu} \stackrel{(+-)}{z}_{[\kappa \lambda]}+ \\
& \left.+4 b_{3} V_{\mu \nu}^{+} \stackrel{[-1]}{z}[\kappa \lambda]_{[\kappa \lambda}+4 b_{3} V_{\mu \nu}^{+} \stackrel{[-2]}{z}[\kappa \lambda]_{[\kappa]}+4 b_{3} V_{\mu \nu}^{-} \stackrel{[+1]}{z}_{[\kappa \lambda]}+4 b_{3} V_{\mu \nu}^{-} \stackrel{[+2]}{z}_{[\kappa \lambda]}\right)
\end{aligned}
$$

Making the same simplifications using the results of the previous sections, we have

$$
\begin{aligned}
& \mathcal{L}_{3}^{\operatorname{spin} A}=4 b_{1} F_{\mu \nu}{ }^{[12]}{ }^{[\mu \nu]}+4 b_{2} U_{\mu \nu}{ }^{[12]}{ }^{[\mu \nu]}+4 b_{1} F_{\mu \nu}{ }^{[+-]^{[}}[\mu \nu]+4 b_{2} U_{\mu \nu}{ }^{[+-]^{[\mu \nu]}}+
\end{aligned}
$$

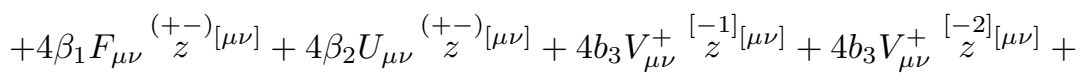

$$
\begin{aligned}
& +4 b_{3} V_{\mu \nu}^{-}{ }_{z}^{[+1]}[\mu \nu]+4 b_{3} V_{\mu \nu}^{-}{ }_{z}^{[+22}[\mu \nu]
\end{aligned}
$$

We can write

$$
\begin{aligned}
& \mathcal{L}_{3}^{\text {spin } A}=\mathcal{L}_{3}^{A S}+\Delta_{3}^{\operatorname{spin}, A} \\
& \Delta_{3}^{\text {spin }, A}=0
\end{aligned}
$$

The spintronics for the triliniear symmetric eq.(3.13) is 


$$
\begin{aligned}
& \mathcal{L}_{3}^{\operatorname{spin} S}=\left(-\frac{1}{2}\left(\Sigma^{\mu \kappa}\right)_{\sigma}^{\rho}\left(\Sigma^{\nu \lambda}\right)_{\rho}^{\sigma}\right)\left(2 \beta_{1} S_{\mu \nu}^{1} \stackrel{(11)}{z}_{\kappa \lambda}+4 \beta_{1} S_{\mu \nu}^{1} \stackrel{(12)}{z}_{\kappa \lambda}+2 \beta_{1} S_{\mu \nu}^{1} \stackrel{(22)}{z}_{\kappa \lambda}+4 \beta_{1} S_{\mu \nu}^{1}{ }^{+} \bar{z}_{\kappa \lambda}^{-3}+\right. \\
& +2 \beta_{2} S_{\mu \nu}^{2} \stackrel{(11)}{z}_{\kappa \lambda}+4 \beta_{2} S_{\mu \nu}^{2} \stackrel{(12)}{z}_{\kappa \lambda}+2 \beta_{2} S_{\mu \nu}^{2} \stackrel{(22)}{z}_{\kappa \lambda}+4 \beta_{2} S_{\mu \nu}^{2}{ }^{+}{ }^{3}{ }_{\kappa \lambda}+ \\
& \left.+4 \beta_{3} S_{\mu \nu}^{+} \stackrel{(-1)}{z}_{\kappa \lambda}+4 \beta_{3} S_{\mu \nu}^{+} \stackrel{(-2)}{z}_{\kappa \lambda}+4 \beta_{3} S_{\mu \nu}^{-} \stackrel{(+1)}{z}_{\kappa \lambda}+4 \beta_{3} S_{\mu \nu}^{-} \stackrel{(+2)}{z} \kappa \lambda_{\kappa \lambda}\right)+ \\
& +\left(-\frac{1}{24}\left(\Sigma_{\kappa \lambda}\right)_{\sigma}^{\rho}\left(\Sigma^{\kappa \lambda}\right)_{\rho}^{\sigma}\right)\left(2 \rho_{1} S_{\mu}^{\mu 1} \stackrel{(11)}{z}_{\nu}+4 \rho_{1} S_{\mu}^{\mu 1} \stackrel{(12)}{z}_{\nu}+2 \rho_{1} S_{\mu}^{\mu 1} \stackrel{(22)}{z}_{\nu}+4 \rho_{1} S_{\mu}^{\mu 1}+{ }_{z}^{-3}{ }_{\nu}+\right. \\
& +2 \rho_{2} S_{\mu}^{\mu 2} \stackrel{(11)}{z}{ }_{\nu}+4 \rho_{2} S_{\mu}^{\mu 2} \stackrel{(12)}{z}_{\nu}+2 \rho_{2} S_{\mu}^{\mu 2} \stackrel{(22)}{z}_{\nu}+4 \rho_{2} S_{\mu}^{\mu 2}{ }_{z}^{+3}{ }_{\nu}+ \\
& +4 \rho_{3} S_{\mu}^{\mu+} \stackrel{(-1)}{z}_{\nu}+4 \rho_{3} S_{\mu}^{\mu+} \stackrel{(-2)}{z}_{\nu}+4 \rho_{3} S_{\mu}^{\mu-} \stackrel{(+1)}{z}_{\nu}+4 \rho_{3} S_{\mu}^{\mu-} \stackrel{(+2)}{z}_{\nu}+ \\
& +2\left(\beta_{1}+4 \rho_{1}\right) S_{\mu}^{\mu 1} \stackrel{(11)}{\omega}{ }_{\nu}+4\left(\beta_{1}+4 \rho_{1}\right) S_{\mu}^{\mu 1} \stackrel{(12)}{\omega}_{\nu}+
\end{aligned}
$$

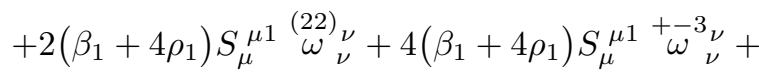

$$
\begin{aligned}
& +2\left(\beta_{2}+4 \rho_{2}\right) S_{\mu}^{\mu 2} \stackrel{(11)}{\omega}_{\nu}+4\left(\beta_{2}+4 \rho_{2}\right) S_{\mu}^{\mu 2} \stackrel{(12)}{\omega}_{\nu}+
\end{aligned}
$$

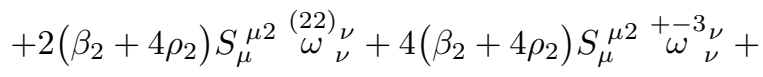

$$
\begin{aligned}
& +4\left(\beta_{3}+4 \rho_{3}\right) S_{\mu}^{\mu+}{\stackrel{(-1)}{\omega}{ }_{\nu}}+4\left(\beta_{3}+4 \rho_{3}\right) S_{\mu}^{\mu+} \stackrel{(-2)}{\omega}_{\nu}+ \\
& +4\left(\beta_{3}+4 \rho_{3}\right) S_{\mu}^{\mu-} \stackrel{(+1)}{\omega}_{\nu}+4\left(\beta_{3}+4 \rho_{3}\right) S_{\mu}^{\mu-}\left(_{\omega}^{(2)} \nu\right)
\end{aligned}
$$


which can be written as

$$
\begin{aligned}
& \mathcal{L}_{3}^{\operatorname{spin} S}=2 \beta_{1} S_{\mu \nu}^{1} \stackrel{(11)}{z} \mu \nu-2 \beta_{1} S_{\mu}^{\mu 1} \stackrel{(11)}{z} \underset{\nu}{\nu}+4 \beta_{1} S_{\mu \nu}^{1} \stackrel{(12)}{z} \mu \nu-4 \beta_{1} S_{\mu}^{\mu 1} \stackrel{(12)}{z}{ }_{\nu}+ \\
& +2 \beta_{1} S_{\mu \nu}^{1} \stackrel{(22)}{z} \mu \nu-2 \beta_{1} S_{\mu}^{\mu 1} \stackrel{(22)}{z} \underset{\nu}{\nu}+4 \beta_{1} S_{\mu \nu}{ }^{+}{ }_{z}^{-3} \mu \nu-4 \beta_{1} S_{\mu}^{\mu 1}+{ }_{z}^{3}{ }_{\nu}+ \\
& +2 \beta_{2} S_{\mu \nu}^{2} \stackrel{(11)}{z} \mu \nu-2 \beta_{2} S_{\mu}^{\mu 2} \stackrel{(11)}{z} \underset{\nu}{\nu}+4 \beta_{2} S_{\mu \nu}^{2} \stackrel{(12)}{z} \mu \nu-4 \beta_{2} S_{\mu}^{\mu 2} \stackrel{(12)}{z}{ }_{\nu}^{\nu}+ \\
& +2 \beta_{2} S_{\mu \nu}^{2} \stackrel{(22)}{z} \mu \nu-2 \beta_{2} S_{\mu}^{\mu 2} \stackrel{(22)}{z} \underset{\nu}{\nu}+4 \beta_{2} S_{\mu \nu}^{2}+{ }^{+3} \mu \nu-4 \beta_{2} S_{\mu}^{\mu 2}{ }^{+-3}{ }_{\nu}+ \\
& +4 \beta_{3} S_{\mu \nu}^{+}{ }^{(-1)}{ }^{(-1)} \mu \nu+4 \beta_{3} S_{\mu \nu}^{+} \stackrel{(-2)}{z} \mu \nu+4 \beta_{3} S_{\mu \nu}^{-} \stackrel{(+1)}{z} \mu \nu+4 \beta_{3} S_{\mu \nu}^{-} \stackrel{(+2)}{z} \mu \nu+ \\
& -4 \beta_{3} S_{\mu}^{\mu+}{ }_{z}^{(-1)}{ }_{\nu}-4 \beta_{3} S_{\mu}^{\mu+}{ }_{z}^{(-2)} \underset{\nu}{\nu}-4 \beta_{3} S_{\mu}^{\mu-}{ }_{z}^{(+1)} \underset{\nu}{\nu}-4 \beta_{3} S_{\mu}^{\mu-}{ }_{z}^{(+2)}{ }_{\nu}+ \\
& +2 \rho_{1} S_{\mu}^{\mu 1} \stackrel{(11)}{z}_{\nu}+4 \rho_{1} S_{\mu}^{\mu 1} \stackrel{(12)}{z}_{\nu}+2 \rho_{1} S_{\mu}^{\mu 1} \stackrel{(22)}{z}_{\nu}+4 \rho_{1} S_{\mu}^{\mu 1}+{ }_{z}^{-3}{ }_{\nu}+ \\
& +2 \rho_{2} S_{\mu}^{\mu 2} \stackrel{(11)}{z}_{\nu}+4 \rho_{2} S_{\mu}^{\mu 2} \stackrel{(12)}{z}_{\nu}+2 \rho_{2} S_{\mu}^{\mu 2} \stackrel{(22)}{z}_{\nu}+4 \rho_{2} S_{\mu}^{\mu 2}+{ }_{z}{ }_{\nu}{ }_{\nu}+ \\
& +4 \rho_{3} S_{\mu}^{\mu+} \stackrel{(-1)}{z}_{\nu}+4 \rho_{3} S_{\mu}^{\mu+} \stackrel{(-2)}{z}_{\nu}+4 \rho_{3} S_{\mu}^{\mu-} \stackrel{(+1)}{z}_{\nu}+4 \rho_{3} S_{\mu}^{\mu-}{ }_{z}^{(+2)}{ }_{\nu}+ \\
& +2\left(\beta_{1}+4 \rho_{1}\right) S_{\mu}^{\mu 1} \stackrel{(11)}{\omega}_{\nu}+4\left(\beta_{1}+4 \rho_{1}\right) S_{\mu}^{\mu 1} \stackrel{(12)}{\omega}_{\nu}+ \\
& +2\left(\beta_{1}+4 \rho_{1}\right) S_{\mu}^{\mu 1} \stackrel{(22)}{\omega}_{\nu}+4\left(\beta_{1}+4 \rho_{1}\right) S_{\mu}^{\mu 1}{ }^{+-3}{ }_{\nu}+ \\
& +2\left(\beta_{2}+4 \rho_{2}\right) S_{\mu}^{\mu 2} \stackrel{(11)}{\omega}_{\nu}+4\left(\beta_{2}+4 \rho_{2}\right) S_{\mu}^{\mu 2} \stackrel{(12)}{\omega}_{\nu}+ \\
& +2\left(\beta_{2}+4 \rho_{2}\right) S_{\mu}^{\mu 2} \stackrel{(22)}{\omega}_{\nu}^{\nu}+4\left(\beta_{2}+4 \rho_{2}\right) S_{\mu}^{\mu 2}{ }^{+-3}{ }_{\nu}+ \\
& +4\left(\beta_{3}+4 \rho_{3}\right) S_{\mu}^{\mu+} \stackrel{(-1)}{\omega}_{\nu}+4\left(\beta_{3}+4 \rho_{3}\right) S_{\mu}^{\mu+\left(_{\nu}^{-2)}\right.}{ }_{\nu}+ \\
& +4\left(\beta_{3}+4 \rho_{3}\right) S_{\mu}^{\mu-} \stackrel{(+1)}{\omega}_{\nu}+4\left(\beta_{3}+4 \rho_{3}\right) S_{\mu}^{\mu-} \stackrel{(+2)}{\omega}_{\nu}
\end{aligned}
$$

It yields,

$$
\mathcal{L}_{3}^{\operatorname{spin} S}=\mathcal{L}_{3}^{S s}+\Delta_{3}^{\operatorname{spin}, S}
$$

where

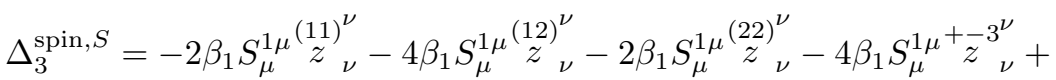

$$
\begin{aligned}
& -2 \beta_{2} S_{\mu}^{2 \mu} \stackrel{(11)}{z}{ }_{\nu}-4 \beta_{2} S_{\mu}^{2 \mu} \stackrel{(12)}{z}{ }_{\nu}-2 \beta_{2} S_{\mu}^{2 \mu} \stackrel{(22)}{z}{ }_{\nu}-4 \beta_{2} S_{\mu}^{2 \mu}+{ }_{z}^{-3}{ }_{\nu}+ \\
& -4 \beta_{3} S_{\mu}^{+\mu} \stackrel{(-1)}{z} \underset{\nu}{\nu}-4 \beta_{3} S_{\mu}^{+\mu} \stackrel{(-2)}{z} \underset{\nu}{\nu}-4 \beta_{3} S_{\mu}^{-\mu} \stackrel{(+2)}{z}_{\nu}^{\nu}-4 \beta_{3} S_{\mu}^{-\mu} \stackrel{(+2)}{z}{ }_{\nu}
\end{aligned}
$$

Notice that eq. (3.42) shows the presence of spin-valued contributions. 
Simplifying, we have

$$
\begin{aligned}
& \mathcal{L}_{3}^{\operatorname{spin} S}=2 \beta_{1} S_{\mu \nu}^{1} \stackrel{(11)}{z} \mu \nu+4 \beta_{1} S_{\mu \nu}^{1} \stackrel{(12)}{z} \mu \nu+2 \beta_{1} S_{\mu \nu}^{1} \stackrel{(22)}{z} \mu \nu+4 \beta_{1} S_{\mu \nu}^{1}{ }^{+}{ }^{-3} \mu \nu+ \\
& +2 \beta_{2} S_{\mu \nu}^{2} \stackrel{(11)}{z} \mu \nu+4 \beta_{2} S_{\mu \nu}^{2} \stackrel{(12)}{z} \mu \nu+2 \beta_{2} S_{\mu \nu}^{2} \stackrel{(22)}{z} \mu \nu+4 \beta_{2} S_{\mu \nu}^{2}{ }^{+} z^{3} \mu \nu+ \\
& +4 \beta_{3} S_{\mu \nu}^{+} \stackrel{(-1)}{z} \mu \nu+4 \beta_{3} S_{\mu \nu}^{+} \stackrel{(-2)}{z} \mu \nu+4 \beta_{3} S_{\mu \nu}^{-} \stackrel{(+1)}{z} \mu \nu+4 \beta_{3} S_{\mu \nu}^{-} \stackrel{(+2)}{z} \mu \nu+ \\
& +2\left(\rho_{1}-\beta_{1}\right) S_{\mu}^{\mu 1} \stackrel{(11)}{z}{ }_{\nu}+4\left(\rho_{1}-\beta_{1}\right) S_{\mu}^{\mu 1} \stackrel{(12)}{z}_{\nu}+ \\
& +2\left(\rho_{1}-\beta_{1}\right) S_{\mu}^{\mu 1} \stackrel{(22)}{z}_{\nu}+4\left(\rho_{1}-\beta_{1}\right) S_{\mu}^{\mu 1}+{ }_{z}{ }_{\nu}^{3}+ \\
& +2\left(\rho_{2}-\beta_{2}\right) S_{\mu}^{\mu 2} \stackrel{(11)}{z}{ }_{\nu}+4\left(\rho_{2}-\beta_{2}\right) S_{\mu}^{\mu 2} \stackrel{(12)}{z}_{\nu}+ \\
& +2\left(\rho_{2}-\beta_{2}\right) S_{\mu}^{\mu 2} \stackrel{(22)}{z}_{\nu}+4\left(\rho_{2}-\beta_{2}\right) S_{\mu}^{\mu 2}+{ }_{z}^{-3}{ }_{\nu}+ \\
& +4\left(\rho_{3}-\beta_{3}\right) S_{\mu}^{\mu+} \stackrel{(-1)}{z} \underset{\nu}{\nu}+4\left(\rho_{3}-\beta_{3}\right) S_{\mu}^{\mu+} \stackrel{(-2)}{z}_{\nu}+
\end{aligned}
$$

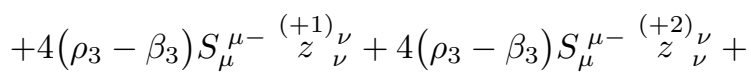

$$
\begin{aligned}
& +2\left(\beta_{1}+4 \rho_{1}\right) S_{\mu}^{\mu 1} \stackrel{(11)}{\omega}{ }_{\nu}+4\left(\beta_{1}+4 \rho_{1}\right) S_{\mu}^{\mu 1} \stackrel{(12)}{\omega}_{\nu}+
\end{aligned}
$$

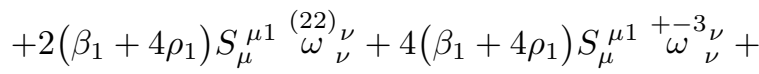

$$
\begin{aligned}
& +2\left(\beta_{2}+4 \rho_{2}\right) S_{\mu}^{\mu 2} \stackrel{(11)}{\omega}_{\nu}+4\left(\beta_{2}+4 \rho_{2}\right) S_{\mu}^{\mu 2} \stackrel{(12)}{\omega}_{\nu}+ \\
& +2\left(\beta_{2}+4 \rho_{2}\right) S_{\mu}^{\mu 2} \stackrel{(22)}{\omega}_{\nu}+4\left(\beta_{2}+4 \rho_{2}\right) S_{\mu}^{\mu 2}{ }^{+-3}{ }_{\nu}+ \\
& +4\left(\beta_{3}+4 \rho_{3}\right) S_{\mu}^{\mu+} \stackrel{(-1)}{\omega}_{\nu}+4\left(\beta_{3}+4 \rho_{3}\right) S_{\mu}^{\mu+} \stackrel{(-2)}{\omega}_{\nu}+ \\
& +4\left(\beta_{3}+4 \rho_{3}\right) S_{\mu}^{\mu-} \stackrel{(+1)}{\omega}_{\nu}+4\left(\beta_{3}+4 \rho_{3}\right) S_{\mu}^{\mu-} \stackrel{(+2)}{\omega}_{\nu}
\end{aligned}
$$

Considering the $\mathcal{L}_{3}^{\text {spin st }}$ tri-valued semi-topological term, one gets

$$
\begin{aligned}
& \mathcal{L}_{3}^{\text {spinst }}=\left(-\frac{1}{24}\left(\Sigma^{\mu \nu}\right)_{\sigma}^{\rho}\left(\Sigma_{\mu \nu}\right)_{\rho}^{\sigma}\right) \epsilon^{\mu \nu \rho \sigma}\left\{2 i \stackrel{[1+]}{z}_{\mu \nu} V_{\rho \sigma}^{-}-2 i \stackrel{[1-]}{z}_{\mu \nu} V_{\rho \sigma}^{+}+\right. \\
& \left.+2 i \stackrel{[2+]}{z}_{\mu \nu} V_{\rho \sigma}^{-}-2 i \stackrel{[2-]}{z}_{\mu \nu} V_{\rho \sigma}^{+}+\left(\alpha_{0} F_{\rho \sigma}+\alpha_{1} U_{\rho \sigma}\right)\left[i\left(\stackrel{[+-]}{z}_{\mu \nu}+\stackrel{[-+]}{z}_{\mu \nu}\right)\right]+4 \stackrel{[12]}{z}_{\mu \nu}\left(\alpha_{0} F_{\rho \sigma}+\alpha_{1} U_{\rho \sigma}\right)\right\} .
\end{aligned}
$$

where we have

$$
\begin{aligned}
& \stackrel{[1 \pm]}{z}_{\mu \nu}=\alpha_{0}\left(A_{\mu} V_{\nu}^{ \pm}-A_{\nu} V_{\mu}^{ \pm}\right) \\
& \stackrel{[2 \pm]}{z}_{\mu \nu}=\alpha_{1}\left(U_{\mu} V_{\nu}^{ \pm}-U_{\nu} V_{\mu}^{ \pm}\right)
\end{aligned}
$$

It yields, 


$$
\begin{aligned}
& \mathcal{L}_{3}^{\text {spinst }}=\mathcal{L}_{3}^{\text {st }}+\Delta_{3}^{\text {spin }, s t} \\
& \Delta_{3}^{\text {spin }, s t}=0
\end{aligned}
$$

A further development is on the quadrilinear terms at eq. (3.15). Starting with $\mathcal{L}_{4}^{\text {spin } A}$ : quadrilinear antisymmetric term, one gets

$$
\mathcal{L}_{4}^{\operatorname{spin} A}=\left(\frac{1}{4}\left(\Sigma^{\mu \nu}\right)_{\sigma}^{\rho}\left(\Sigma^{\kappa \lambda}\right)_{\rho}^{\sigma}+\frac{1}{2}\left(\Sigma^{\mu \kappa}\right)_{\sigma}^{\rho}\left(\Sigma^{\nu \lambda}\right)_{\rho}^{\sigma}\right) \mathcal{L}_{4}^{A}
$$

where the term $\mathcal{L}_{4}^{A}$ is defined at eq.(3.16). Simplifying, we have

$$
\begin{aligned}
& \mathcal{L}_{4}^{\operatorname{spin} A}=\mathcal{L}_{4}^{A}+\Delta_{4}^{\mathrm{spin}, A} \\
& \Delta_{4}^{\mathrm{spin}, A}=0
\end{aligned}
$$


For the symmetric tensor, eq. (3.17) gives $\mathcal{L}_{4}^{\text {spin } S}$,

$$
\begin{aligned}
& \mathcal{L}_{4}^{\operatorname{spin} S}=\frac{1}{2}\left(\Sigma^{\mu \kappa}\right)_{\sigma}^{\rho}\left(\Sigma^{\nu \lambda}\right)_{\rho}^{\sigma}\left(2 \stackrel{[12]}{z}_{(\mu \nu)} \stackrel{[12]}{z}_{(\kappa \lambda)}+2 \stackrel{[12]}{z}_{(\mu \nu)} \stackrel{[21]}{z}_{(\kappa \lambda)}+4 \stackrel{[13+]}{z}_{(\mu \nu)}^{[13-]} z_{(\kappa \lambda)}+\right.
\end{aligned}
$$

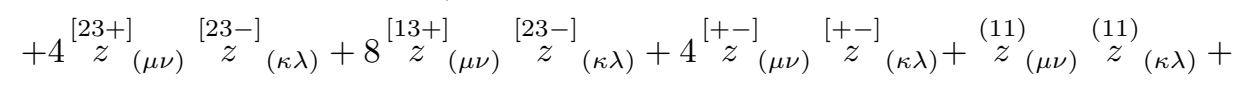

$$
\begin{aligned}
& +\stackrel{(22)}{z}_{(\mu \nu)} \stackrel{(22)}{z}_{(\kappa \lambda)}+2 \stackrel{(11)}{z}_{(\mu \nu)} \stackrel{(22)}{z}_{(\kappa \lambda)}+4 \stackrel{(12)}{z}_{(\mu \nu)} \stackrel{(12)}{z}_{(\kappa \lambda)}+2 \stackrel{(11)}{z}_{(\mu \nu)} \stackrel{(12)}{z}_{(\kappa \lambda)}+ \\
& +4 \stackrel{(22)}{z}_{(\mu \nu)} \stackrel{(12)}{z}_{(\kappa \lambda)}+4\left(\stackrel{(11)}{z}_{(\mu \nu)}+\stackrel{(22)}{z}_{(\mu \nu)}\right){ }^{+-3}{ }_{(\kappa \lambda)}+4 \stackrel{(11)}{\omega}_{(\mu \nu)} \stackrel{(11)}{\omega}_{(\kappa \lambda)}+ \\
& +4 \stackrel{(22)}{\omega}_{(\mu \nu)} \stackrel{(22)}{\omega}_{(\kappa \lambda)}+16 \stackrel{(11)}{\omega}_{(\mu \nu)} \stackrel{(12)}{\omega}_{(\kappa \lambda)}+16 \stackrel{(22)}{\omega}_{(\mu \nu)} \stackrel{(12)}{\omega}_{(\kappa \lambda)}+16 \stackrel{(12)}{\omega}_{(\mu \nu)} \stackrel{(12)}{\omega}_{(\kappa \lambda)}+ \\
& +2 \stackrel{(11)}{z}_{(\mu \nu)} \stackrel{(11)}{\omega}_{(\kappa \lambda)}+2 \stackrel{(22)}{z}_{(\mu \nu)} \stackrel{(22)}{\omega}_{(\kappa \lambda)}+8 \stackrel{(12)}{z}_{(\mu \nu)} \stackrel{(11)}{\omega}_{(\kappa \lambda)}+8 \stackrel{(12)}{z}_{(\mu \nu)} \stackrel{(22)}{\omega}_{(\kappa \lambda)}+
\end{aligned}
$$

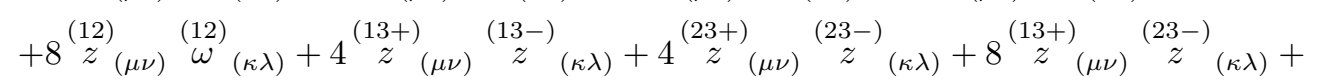

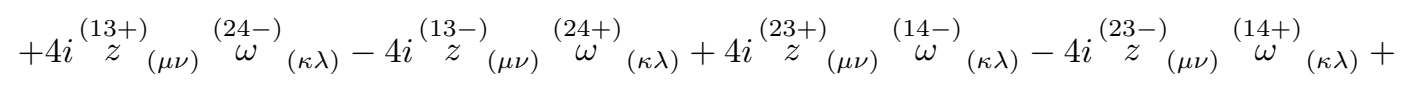

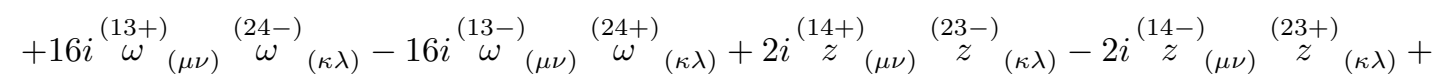

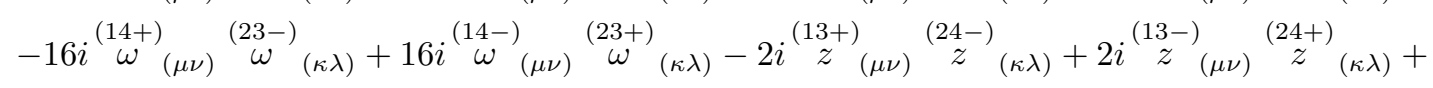

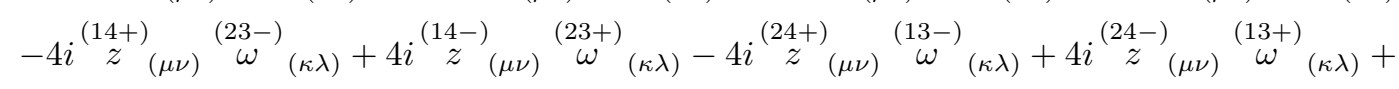

$$
\begin{aligned}
& \left.+8 \stackrel{(12)}{z}(\mu \nu)^{+-3}{ }_{(\kappa \lambda)}+4{ }^{+-3}(\mu \nu)+z_{(\kappa \lambda)}\right)+
\end{aligned}
$$

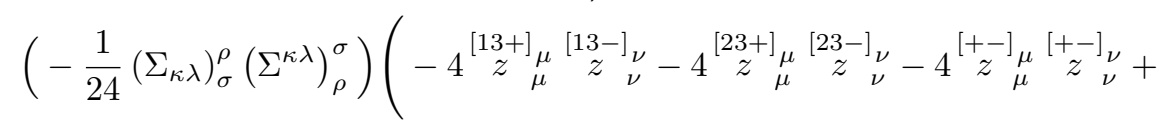

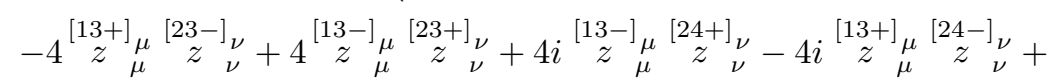

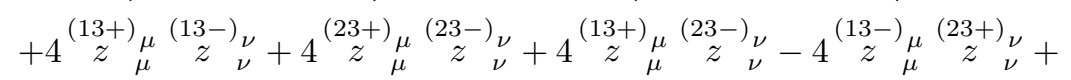

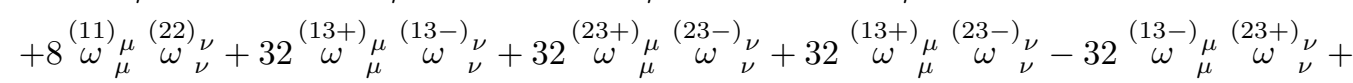

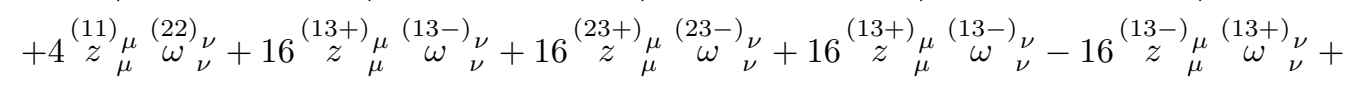

$$
\begin{aligned}
& +8\left\{\stackrel{(11)}{z}_{\mu}^{\mu}+\stackrel{(22)}{z}_{\mu}+2 \stackrel{(11)}{\omega}_{\mu}^{\mu}+2 \stackrel{(22)}{\omega}_{\mu}^{\mu}+2 \stackrel{(12)}{z}_{\mu}^{\mu}+4 \stackrel{(12)}{\omega}_{\mu}^{\mu}\right\}{ }^{+-3}{ }_{\nu}^{\nu}+
\end{aligned}
$$

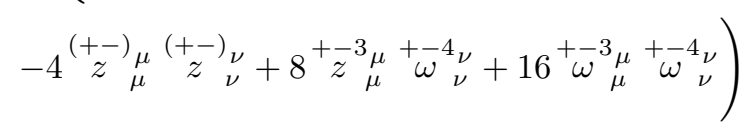


Using the relations in the previous section, we have

$$
\mathcal{L}_{4}^{\operatorname{spin} S}=\mathcal{L}_{4}^{S}+\Delta_{4}^{\text {spin }, S}
$$

where

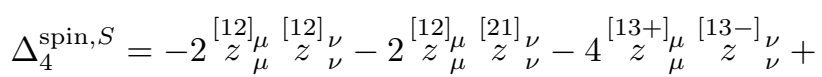

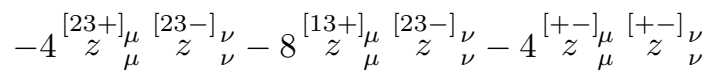

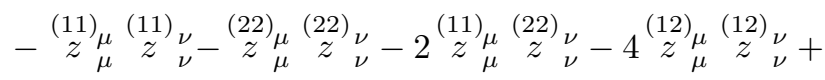

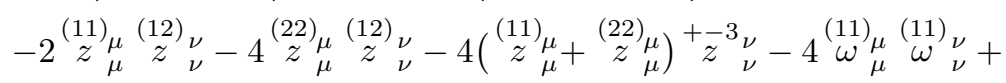

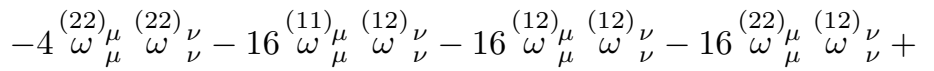

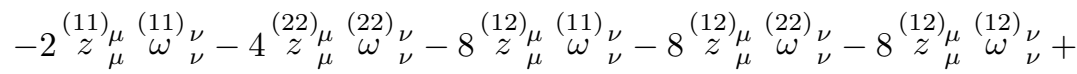

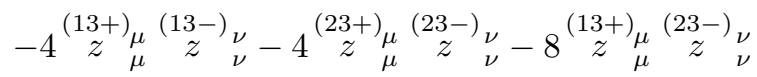

$$
\begin{aligned}
& \left.\left.-4 i{ }_{z}^{(13+)}\right)_{\mu} \stackrel{(24-)}{\omega} \nu_{\nu}+4 i{ }_{z}^{(13-)}\right)_{\mu} \stackrel{(24+)}{\omega} \nu_{\nu}-4 i{ }_{z}^{(23+)_{\mu}}{\left.\stackrel{(14-)}{\omega})_{\nu}+4 i{ }_{z}^{(23-)}\right)_{\mu}(14+)}_{\omega}^{\nu}+
\end{aligned}
$$

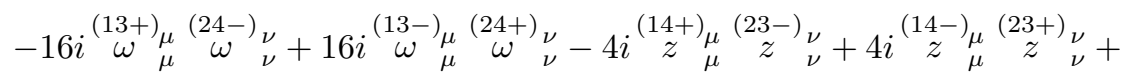

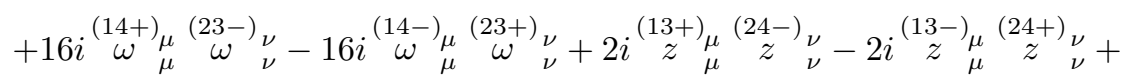

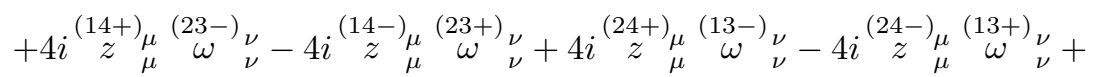

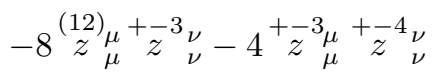

Eq. (3.52) is showing on eq.(2.5) contribution.

Finally, for $\mathcal{L}_{4}^{\text {spinst }}$, one gets

$$
\begin{aligned}
& \mathcal{L}_{4}^{s t}=\left(-\frac{1}{24}\left(\Sigma^{\mu \nu}\right)_{\sigma}^{\rho}\left(\Sigma_{\mu \nu}\right)_{\rho}^{\sigma}\right) \epsilon^{\mu \nu \rho \sigma}\left\{4 \stackrel{[12]}{z}_{\mu \nu}^{[12]}{ }_{\rho \sigma}+\right. \\
& \left.+4 \stackrel{[12]}{z}_{\mu \nu}\left(\stackrel{[+-]}{z}_{\rho \sigma}+\stackrel{[-+]}{z}_{\rho \sigma}\right)+\left(\stackrel{[+-]}{z}_{\mu \nu}+\stackrel{[-+]}{z}_{\mu \nu}\right)\left({ }^{[+-]}{ }_{\rho \sigma}+\stackrel{[-+]}{z}_{\rho \sigma}\right)\right\} .
\end{aligned}
$$

Using the eq.(3.18) in the previous section, we have

$$
\mathcal{L}_{4}^{\text {spin st }}=\mathcal{L}_{4}^{\text {st }}+\Delta_{4}^{\text {spinst }}
$$


and

$$
\Delta_{4}^{\text {spinst }}=0
$$

Summarizing, by considering fields Lorentz algebra value eq.(2.5), one gets a Spin-Valued Four Bosons Lagrangian given by

$$
\mathcal{L}^{\text {spin }}=\mathcal{L}_{\text {Four EM }}+\triangle \mathcal{L}_{\text {algebra valued }}
$$

where

$$
\triangle \mathcal{L}_{\text {algebra valued }}^{s}=\triangle_{3}^{\mathrm{spin}, S}+\triangle_{4}^{\mathrm{spin}, S}
$$

showing that only trilinear and quadrilinear symmetric terms receive spin contributions, as eqs.(3.43) and eq.(3.53) are showing.

\section{Equations of Motion}

Eq.(3.56) provides a spin-valued antireductionist nonlinear abelian Lagrangian. Its hyperbolic system of equations of motion contain spin dependence, propagates granular and collective fields, massive terms and with nonlinear sources. The I-equation of motion to each field $A_{\mu I}$ has the following general expression:

$$
\partial_{\nu}\left(F_{I}^{\nu \mu}+z_{I}^{[\nu \mu]}\right)+\partial^{\mu}\left(S_{\alpha I}^{\alpha}+z_{\alpha I}^{\alpha}\right)+M_{I}^{\mu}+l_{I}^{\mu}+c_{I}^{\mu}=j_{I}^{\mu}
$$

Both spin dynamics are splitted by multiplying eq.(4.1) by the longitudinal and transversal operators. For this, we must take the operators $A_{\mu I}^{T}=\theta_{\mu \nu} A_{I}^{\nu}$ and $A_{\mu I}^{L}=\omega_{\mu \nu} A_{I}^{\nu}$ where $\theta_{\mu \nu}=\eta_{\mu \nu}-\frac{\partial_{\mu} \partial_{\nu}}{\square}$ and $\omega_{\mu \nu}=\frac{\partial_{\mu} \partial_{\nu}}{\square}$.

For spin-1 dynamics:

$$
\partial_{\nu}\left(F_{I}^{\nu \mu}+z_{I}^{[\nu \mu]}\right)+M_{T I}^{\mu}+l_{T I}^{\mu}+c_{T I}^{\mu}=j_{T I}^{\mu}
$$

For spin-0 dynamics: 


$$
\partial^{\mu}\left(S_{\alpha I}^{\alpha}+z_{\alpha I}^{\alpha}\right)+M_{L I}^{\mu}+l_{L I}^{\mu}+c_{L I}^{\mu}=j_{L I}^{\mu}
$$

where $j_{T I}^{\mu} \equiv \Theta_{\nu}^{\mu} j^{\nu}$ e $j_{L I}^{\mu} \equiv \omega_{\nu}^{\mu} j^{\nu}$ and so forth.

Considering that covariance is preserved the separation of spin- 1 and spin-0 sectors is valid. Eqs.(4.2-3) can be considered as physical laws due to the fact that symmetry is mainteined. Thus, the physical continuity equation for spin-1 is

$$
\text { ว. }\left(m^{2} X_{T}^{\mu}+l_{T}^{\mu}+c_{T}^{\mu}-j_{T}^{\mu}\right)=0
$$

and the spin-0 wave equation is

$$
\square\left(S_{\alpha}^{\alpha}+z_{\alpha}^{\alpha}\right)=\partial \cdot\left(m^{2} X_{L}^{\mu}+l_{L}^{\mu}+c_{T}^{\mu}-j_{L}^{\mu}\right)
$$

We should study now such coupled fields equations in terms of the fields quadruplet. Explicit their ingredients.

\section{$5 \quad$ Fields dynamics}

After the generic study on the spin-valued systemic relativistic equations driving the model, we have to make explicit these equations of motion for each field in the quadruplet. For field $A_{\mu}$ (photon), one gets

$A_{\mu}$-spin-1:

$$
\partial_{\nu}\left(F_{A}^{\nu \mu}+z_{A}^{[\nu \mu]}\right)+l_{A T}^{\mu}+c_{A T}^{\mu}=j_{A T}^{\mu}
$$

$A_{\mu}$-spin-0:

$$
\partial^{\mu}\left(S_{\alpha A}^{\alpha}+z_{\alpha A}^{\alpha}\right)+l_{A L}^{\mu}+c_{A L}^{\mu}=j_{A L}^{\mu}
$$

where the antisymmetric sector is given by:

$$
\begin{aligned}
& F_{A}^{\nu \mu}=\bar{a}_{1} F^{\nu \mu} \text { (A-antisymmetric granular), } \\
& \left.z_{A}^{[\nu \mu]}=\bar{a}_{2}\left({ }^{[12]^{[\nu \mu]}}+{ }^{[+-}\right]^{[\nu \mu]}\right)+\bar{a}_{3}{ }_{z}^{(+-)}[\nu \mu] \text { (A-antisymmetric collective) }
\end{aligned}
$$


and the longitudinal term is

$$
\begin{aligned}
& S_{\alpha A}^{\alpha}=\left(\bar{a}_{4} S_{\alpha}^{\alpha 1}+\bar{a}_{5} S_{\alpha}^{\alpha 2}\right) \text { (A-symmetric granular) }
\end{aligned}
$$

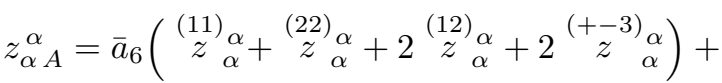

$$
\begin{aligned}
& +\bar{a}_{7}\left(\left(^{(11)}{ }_{\alpha}+\stackrel{(22)}{\omega}_{\alpha} \alpha+2 \stackrel{(12)}{\omega}_{\alpha}^{\alpha}+2{ }^{+} \bar{\omega}_{\alpha}^{-3} \alpha\right)\right. \text { (A-symmetric collective) }
\end{aligned}
$$

The so-called London term is represented by $l_{A}^{\mu}$.

$$
\begin{aligned}
& l_{A}^{\mu}=\left(\Sigma_{\alpha}^{\mu}\right)_{\beta \nu}\left\{\left(f_{2}^{A} \stackrel{(11)}{z}(\alpha \beta)+f_{3}^{A} \stackrel{(12)}{z}(\alpha \beta)_{(\alpha)} f_{2}^{A} \stackrel{(22)}{z}(\alpha \beta)+f_{4}^{A} \stackrel{(11)}{\omega}(\alpha \beta)+f_{5}^{A} \stackrel{(12)}{\omega}(\alpha \beta)+\right.\right.
\end{aligned}
$$

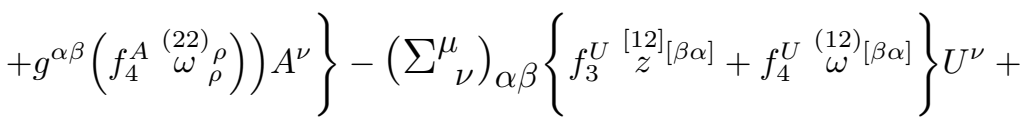

$$
\begin{aligned}
& +\left(\Sigma^{\mu}{ }_{\alpha}\right)_{\beta \nu}\left\{f_{6}^{U} \stackrel{(11)}{z}(\beta \alpha)+f_{7}^{U} \stackrel{(12)}{z}(\beta \alpha)^{(\beta)} f_{8}^{U} \stackrel{(22)}{z}(\beta \alpha)+f_{9}^{U} \stackrel{(11)}{\omega}(\beta \alpha)+f_{4}^{U} \stackrel{(12)}{\omega}(\beta \alpha)+f_{10}^{U} \stackrel{(22)}{\omega}(\beta \alpha)\right\} U^{\nu}
\end{aligned}
$$

The conglomerate term $c_{A}^{\mu}$ is

$$
\begin{aligned}
& c_{A}^{\mu}=\left(\Sigma_{\alpha}^{\mu}\right)_{\beta \nu}\left\{f_{3}^{A}{ }_{z}^{+-3(\beta \alpha)}+g^{\beta \alpha}\left(f_{4}^{A}{ }^{+-3} \rho\right)\right\} A^{\nu}+f_{3}^{U}\left(\Sigma^{\mu}{ }_{\nu}\right)_{\alpha \beta}{ }^{[+-]}{ }^{[\beta \alpha]} U^{\nu}+ \\
& +\left(\Sigma_{\alpha}^{\mu}\right)_{\beta \nu}\left\{f_{7}^{U}{ }^{+} \bar{z}^{3}(\beta \alpha)+g^{\beta \alpha}\left(f_{13}^{U}{ }^{+} \bar{\omega}_{\rho}^{3} \rho\right)\right\} U^{\nu}+
\end{aligned}
$$

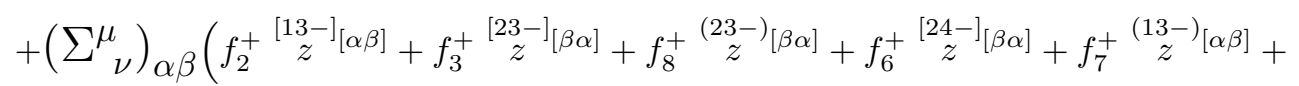

$$
\begin{aligned}
& \left.\left.+f_{11}^{+} \stackrel{(23-)}{\omega}\right)^{[\beta \alpha]}+f_{12}^{+} \stackrel{(24-)}{\omega}{ }^{[\beta \alpha]}\right) V^{+\nu}+ \\
& +\left(\Sigma^{\mu}{ }_{\alpha}\right)_{\beta \nu}\left\{f_{5}^{+} \stackrel{[23-]}{z}(\beta \alpha)+f_{9}^{+} \stackrel{(23-)}{z}(\beta \alpha)+f_{10}^{+} \stackrel{(24-)}{z}(\beta \alpha)+f_{6}^{+} \stackrel{[24-]}{z}(\beta \alpha)+f_{7}^{+} \stackrel{(13-)}{z}(\alpha \beta)+\right. \\
& +f_{11}^{+} \stackrel{(23-)}{\omega}^{(\beta \alpha)}+f_{12}^{+} \stackrel{(24-)}{\omega}^{(\beta \alpha)}+ \\
& \left.+g^{\beta \alpha}\left(f_{14}^{+} \stackrel{[13-]}{z} \underset{\rho}{\rho}+f_{14}^{+} \stackrel{[23-]}{z} \underset{\rho}{\rho}+f_{7}^{+} \stackrel{(13-)}{z} \underset{\rho}{\rho}+f_{7}^{+} \stackrel{(23-)}{z} \underset{\rho}{\rho}+f_{15}^{+} \stackrel{(13-)}{\omega} \underset{\rho}{\rho}+f_{15}^{+} \stackrel{(23-)}{\omega} \underset{\rho}{\rho}\right)\right\} V^{+\nu}+
\end{aligned}
$$

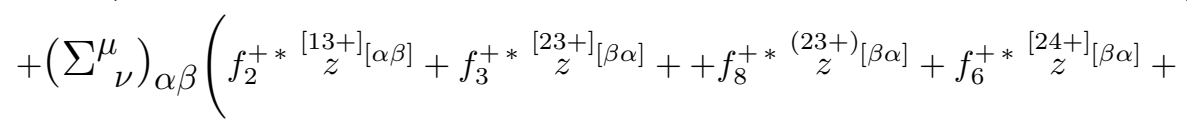

$$
\begin{aligned}
& \left.+f_{7}^{+*} \stackrel{(13+)}{z}[\alpha \beta]^{\left[-f_{11}^{+*}\right.} \stackrel{(23+)}{\omega}[\beta \alpha]+f_{12}^{+*} \stackrel{(24+)}{\omega}[\beta \alpha]\right) V^{-\nu} \\
& +\left(\Sigma_{\alpha}^{\mu}\right)_{\beta \nu}\left\{f_{5}^{+*} \stackrel{[23+]}{z}(\beta \alpha) f_{6}^{+*} \stackrel{[24+]}{z}(\beta \alpha)+f_{7}^{+*} \stackrel{(13+)}{z}(\alpha \beta)+\right. \\
& +f_{9}^{+*} \stackrel{(23+)}{z}(\beta \alpha)+f_{10}^{+*} \stackrel{(24+)}{z}(\beta \alpha)+f_{11}^{+*} \stackrel{(23+)}{\omega}(\beta \alpha)+f_{12}^{+*} \stackrel{(24+)}{\omega}(\beta \alpha)+ \\
& \left.+g^{\beta \alpha}\left(f_{14}^{+*} \stackrel{[13+]}{z} \rho_{\rho}+f_{14}^{+*} \stackrel{[23+]}{\rho} \rho_{\rho}+f_{7}^{+*}{ }_{z}^{(13+)} \rho+f_{7}^{+*} \stackrel{(23+)}{\rho} \rho_{\rho}+f_{15}^{+*} \stackrel{(13+)}{\omega}_{\rho}+f_{15}^{+*} \stackrel{(23+)}{\omega}_{\rho}\right)\right\} V^{-\nu}
\end{aligned}
$$

The non-linear current is 


$$
\begin{aligned}
& j_{A}^{\mu}=\left(\Sigma_{\alpha}^{\mu}{ }_{\beta \nu}\left\{f_{1}^{A} S^{\beta \alpha 2}+g^{\beta \alpha}\left(f_{6}^{A} S_{\rho}^{\rho 1}+f_{7}^{A} S_{\rho}^{\rho 2}\right)\right\} A^{\nu}+\right. \\
& +\left(\Sigma^{\mu}{ }_{\nu}\right)_{\alpha \beta}\left(f_{1}^{U} F^{\beta \alpha}+f_{2}^{U} U^{\beta \alpha}\right) U^{\nu}+ \\
& +\left(\Sigma^{\mu}{ }_{\alpha}\right)_{\beta \nu}\left\{f_{5}^{U} S^{\beta \alpha 2}+g^{\beta \alpha}\left(f_{11}^{U} S_{\rho}^{\rho 1}\right)\right\} U^{\nu}+ \\
& +\left(\Sigma^{\mu}{ }_{\nu}\right)_{\alpha \beta}\left(f_{1}^{+} V^{\beta \alpha-}\right) V^{+\nu}+\left(\Sigma^{\mu}{ }_{\alpha}\right)_{\beta \nu}\left(f_{4}^{+} S^{\beta \alpha-}+g^{\nu \mu}\left(f_{13}^{+} S_{\rho}^{\rho-}\right)\right) V_{\nu}^{+}+ \\
& +\left(\Sigma^{\mu}{ }_{\nu}\right)_{\alpha \beta}\left(f_{1}^{+*} V^{\beta \alpha+}\right) V^{-\nu}+\left(\Sigma^{\mu}{ }_{\alpha}\right)_{\beta \nu}\left(f_{4}^{+*} S^{\beta \alpha+}+g^{\beta \alpha}\left(f_{13}^{+*} S_{\rho}^{\rho+}\right)\right) V_{\nu}^{-}+
\end{aligned}
$$

where the parameters $\bar{a}_{1}, \ldots f_{15}^{+*}$ are defined in Appendix A.

The continuity equation is

$$
\square\left(S_{\alpha A}^{\alpha}+z_{\alpha A}^{\alpha}\right)=\partial \cdot\left(j_{A}+l_{A}+c_{A}\right)
$$

The corresponding expressions of the electromagnetic waves are:

$A_{\mu}$-spin-1:

$$
\square\left(F_{\nu \mu}^{A}+z_{[\nu \mu]}^{A}\right)=\partial_{\mu} d_{\nu}^{A}-\partial_{\nu} d_{\mu}^{A}+\partial^{\alpha} f_{\alpha \mu \nu}^{A}
$$

where

$$
d_{\mu}^{A}=l_{\mu}^{A T}+c_{\mu}^{A}+j_{\mu}^{A T} \quad \text { e } \quad f_{\alpha \mu \nu}^{A}=\bar{a}_{2}\left(f_{\alpha \mu \nu}^{[12]}+f_{\alpha \mu \nu}^{[+-]}\right)+\bar{a}_{3} f_{\alpha \mu \nu}^{(+-)}
$$

$A_{\mu}$-spin-0:

$$
\square\left(S_{\alpha A}^{\alpha}+z_{\alpha A}^{\alpha}\right)=\partial .\left(j_{A}-l_{A}-c_{A}\right)
$$

The next field corresponding to charge exchange is a massive photon. It yields,

$U_{\mu}$-spin-1:

$$
\partial_{\nu}\left(F_{U}^{\nu \mu}+z_{U}^{[\nu \mu]}\right)+M_{U T}^{\mu}+c_{U T}^{\mu}+l_{U T}^{\mu}=j_{U T}^{\mu}
$$


$U_{\mu}$-spin-0:

$$
\partial^{\mu}\left(S_{\alpha U}^{\alpha}+z_{\alpha U}^{\alpha}\right)+M_{U L}^{\mu}+c_{U L}^{\mu}+l_{U L}^{\mu}=j_{U L}^{\mu}
$$

Sector T:

$$
\begin{aligned}
& F_{U}^{\nu \mu}=\bar{b}_{1} U^{\nu \mu}(\mathrm{U} \text {-antisymmetric granular) } \\
& z_{U}^{[\nu \mu]}=\bar{b}_{2}\left({ }^{[12]}\left[{ }^{[\nu \mu]}+{ }^{[+-}\right]^{[\nu \mu]}\right)+\bar{b}_{3}{ }^{(+-)}{ }^{[\nu \mu]} \text { (U-antisymmetric collective) }
\end{aligned}
$$

Sector L:

$$
\begin{aligned}
& S_{U}^{\nu \mu}=\bar{b}_{4} S_{\alpha}^{\alpha 1}+\bar{b}_{5} S_{\alpha}^{\alpha 2} \text { (U-symmetric granular) }
\end{aligned}
$$

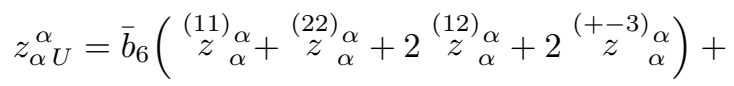

$$
\begin{aligned}
& +\bar{b}_{7}\left(\left(^{(11)}{ }_{\alpha}+\stackrel{(22)}{\omega}_{\alpha}^{\alpha}+2 \stackrel{(12)}{\omega}_{\alpha}^{\alpha}+2{ }^{+-3}{ }^{3} \alpha\right)\right. \text { (U-symmetric colletive) }
\end{aligned}
$$

Sector Mass:

$$
M_{U}^{\mu}=m_{U}^{2} U^{\mu}
$$

Sector-London:

$$
\begin{aligned}
& l_{U}^{\mu}=\left(\Sigma^{\mu}{ }_{\nu}\right)_{\alpha \beta}\left(g_{3}^{A} \stackrel{[12]}{z}[\beta \alpha]+g_{4}^{A} \stackrel{(12)}{\omega}[\beta \alpha]\right) A^{\nu}+ \\
& +\left(\Sigma^{\mu}{ }_{\alpha}\right)_{\beta \nu}\left\{g_{6}^{A} \stackrel{(11)}{z}(\beta \alpha)+g_{7}^{A} \stackrel{(12)}{z}(\beta \alpha)+g_{8}^{A} \stackrel{(22)}{z}(\beta \alpha)+g_{9}^{A} \stackrel{(11)}{\omega}(\beta \alpha)+g_{4}^{A} \stackrel{(12)}{\omega}(\beta \alpha)+g_{10}^{A} \stackrel{(22)}{\omega}(\beta \alpha)\right\} A^{\nu}+ \\
& +\left(\Sigma^{\mu}{ }_{\alpha}\right)_{\beta \nu}\left\{g_{2}^{U} \stackrel{(11)}{z}(\beta \alpha)+2 g_{2}^{U} \stackrel{(12)}{z}(\beta \alpha)+g_{2}^{U} \stackrel{(22)}{z}(\beta \alpha)+g_{3}^{U} \stackrel{(12)}{\omega}(\beta \alpha)+2 g_{3}^{U} \stackrel{(22)}{\omega}(\beta \alpha)+g^{\beta \alpha}\left(g_{3}^{U} \stackrel{11}{\omega} \rho_{\rho}^{\rho}\right)\right\} U^{\nu}
\end{aligned}
$$

Sector-conglomerate:

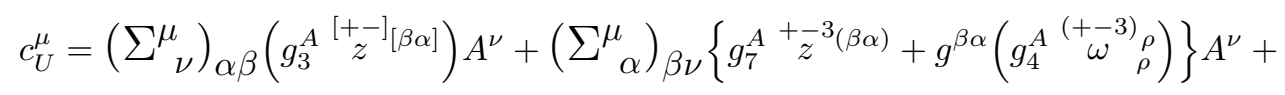

$$
\begin{aligned}
& +\left(\Sigma_{\alpha}^{\mu}\right)_{\beta \nu}\left\{2 g_{2}^{U}{ }^{+-3} z^{(\nu \mu)}+2 g_{3}^{U}{ }^{+-3}{ }_{\rho}^{-3}\right\} U^{\nu}+ \\
& +\left(\Sigma^{\mu}{ }_{\nu}\right)_{\alpha \beta}\left(g_{2}^{+} \stackrel{[13-]}{z}[\alpha \beta]+g_{3}^{+} \stackrel{[14-]^{[\beta \alpha]}}{ }+g_{4}^{+} \stackrel{[23-]}{z}[\alpha \beta]+g_{8}^{+} \stackrel{(23-)}{z}[\alpha \beta]+g_{9}^{+} \stackrel{(13-)}{\omega}[\beta \alpha]\right) V^{+\nu}+ \\
& +\left(\Sigma^{\mu}{ }_{\alpha}\right)_{\beta \nu}\left(g_{2}^{+} \stackrel{[13-]}{z}(\alpha \beta)+g_{3}^{+} \stackrel{[14-]}{z}(\beta \alpha)+g_{4}^{+} \stackrel{[23-]}{z}(\alpha \beta)+g_{6}^{+} \stackrel{(13-)}{z}(\beta \alpha)+g_{7}^{+} \stackrel{(14-)}{z}(\beta \alpha)+g_{8}^{+} \stackrel{(23-)}{z}(\alpha \beta)+\right. \\
& +g_{9}^{+} \stackrel{(13-)}{\omega}^{(\beta \alpha)}+g_{10}^{+} \stackrel{(14-)}{\omega}^{(\beta \alpha)}+g^{\beta \alpha}\left(-g_{4}^{+} \stackrel{[13-]}{z} \underset{\rho}{\rho}-g_{4}^{+} \stackrel{[23-]}{z} \underset{\rho}{\rho}+g_{8}^{+} \stackrel{(13-)}{z} \underset{\rho}{\rho}+g_{8}^{+} \stackrel{(23-)}{z} \underset{\rho}{\rho}+\right. \\
& \left.\left.+g_{8}^{+} \stackrel{(13-)}{\omega} \underset{\rho}{\rho}+g_{13}^{+} \stackrel{(23-)}{\omega} \underset{\rho}{\rho}\right)\right) V^{+\nu}+
\end{aligned}
$$

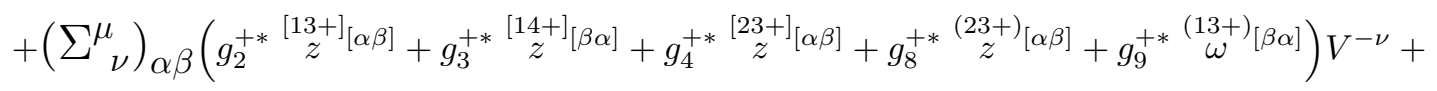

$$
\begin{aligned}
& +\left(\Sigma^{\mu}{ }_{\alpha}\right)_{\beta \nu}\left(g_{2}^{+*} \stackrel{[13+]}{z}(\alpha \beta)_{\left(g_{3}^{+*} \stackrel{[14+]}{z}(\beta \alpha)\right.}+g_{4}^{+*} \stackrel{[23+]}{z}(\alpha \beta)+g_{6}^{+*} \stackrel{(13+)}{z}(\beta \alpha)+g_{7}^{+*} \stackrel{(14+)}{z}(\beta \alpha)+\right.
\end{aligned}
$$

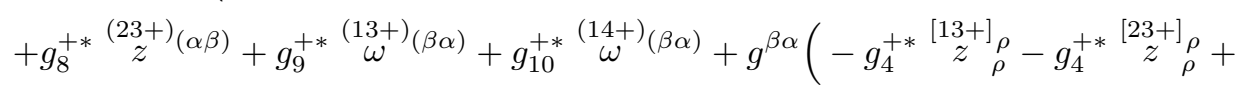

$$
\begin{aligned}
& \left.\left.+g_{8}^{+*}{ }_{z}^{(13+)} \rho_{\rho}+g_{8}^{+*} \stackrel{(23+)}{z} \rho_{\rho}+g_{8}^{+*} \stackrel{(13+)}{\omega}_{\rho}^{\rho}+g_{13}^{+*} \stackrel{(23+)}{\omega}_{\rho}^{\rho}\right)\right) V^{-\nu}
\end{aligned}
$$


Sector current:

$$
\begin{aligned}
& j_{U}^{\mu}=\left(\sum_{\nu}^{\mu}\right)_{\alpha \beta}\left(g_{1}^{A} F^{\beta \alpha}+g_{2}^{A} U^{\beta \alpha}\right) A^{\nu}+\left(\Sigma^{\mu}{ }_{\alpha}\right)_{\beta \nu}\left(g_{5}^{A} S^{\beta \alpha 1}+g^{\beta \alpha}\left(g_{11}^{A} S_{\rho}^{\rho 1}+g_{12}^{A} S_{\rho}^{\rho 2}\right)\right) A^{\nu}+ \\
& +\left(\Sigma^{\mu}{ }_{\alpha}\right)_{\beta \nu}\left(g_{1}^{U} S^{\beta \alpha 1}+g^{\beta \alpha}\left(g_{4}^{U} S_{\rho}^{\rho 1}+g_{5}^{U} S_{\rho}^{\rho 2}\right)\right) U^{\nu}+ \\
& +\left(\Sigma^{\mu}{ }_{\nu}\right)_{\alpha \beta}\left(g_{1}^{+} V^{\beta \alpha-}\right) V^{+\nu}+\left(\sum^{\mu}{ }_{\alpha \nu}\right)_{\beta \nu}\left(g_{5}^{+} S^{\beta \alpha-}+g^{\beta \alpha}\left(g_{11}^{+} S_{\alpha}^{\alpha-}\right)\right) V^{\nu-}+ \\
& +\left(\sum^{\mu}{ }_{\nu}\right)_{\alpha \beta}\left(g_{1}^{+*} V^{\beta \alpha+}\right) V^{-\nu}+\left(\sum^{\mu}{ }_{\alpha}\right)_{\beta \nu}\left(g_{5}^{+*} S^{\beta \alpha+}+g^{\beta \alpha}\left(g_{11}^{+*} S_{\alpha}^{\alpha+}\right)\right) V^{+\nu}
\end{aligned}
$$

The corresponding equation of continuity is

$$
\square\left(S_{\alpha U}^{\alpha}+z_{\alpha U}^{\alpha}\right)=\partial \cdot\left(j_{U}+l_{U}+c_{U}+M_{U}\right)
$$

where the parameters $\bar{b}_{1}, \ldots$ are defined in Appendix B.

The corresponding electromagnetic waves expressions are

$U_{\mu}$-spin-1:

$$
\square\left(F_{\nu \mu}^{U}+z_{[\nu \mu]}^{U}\right)=\partial_{\mu} d_{\nu}^{U}-\partial_{\nu} d_{\mu}^{U}+\partial_{\mu} M_{\nu}^{U}-\partial_{\nu} M_{\mu}^{U}+\partial^{\alpha} f_{\alpha \mu \nu}^{U}
$$

where

$$
d_{\mu}^{U}=l_{\mu}^{U T}+c_{\mu}^{U}+j_{\mu}^{U T} \quad \text { e } \quad f_{\alpha \mu \nu}^{U}=\bar{b}_{2}\left(f_{\alpha \mu \nu}^{[12]}+f_{\alpha \mu \nu}^{[+-]}\right)+\bar{b}_{3} f_{\alpha \mu \nu}^{(+-)}
$$

$U_{\mu}$-spin-0:

$$
\square\left(S_{\alpha U}^{\alpha}+z_{\alpha U}^{\alpha}\right)=\partial \cdot\left(j_{U}-l_{U}-c_{U}\right)
$$

Another two fields responsible to the microscopic electromagnetism are the charged fields. For the charged field $V_{\mu}^{+}$ (Massive Photon with positive charge), one gets

$V_{\mu}^{+}$-spin-1:

$$
\partial_{\nu}\left(F_{+}^{\nu \mu}+z_{+}^{[\nu \mu]}\right)+M_{+T}^{\mu}+l_{+T}^{\mu}+c_{+T}^{\mu}=j_{V^{+} T}^{\mu}
$$

$V_{\mu}^{+}$-spin-0:

$$
\partial^{\mu}\left(S_{\alpha+}^{\alpha}+z_{\alpha+}^{\alpha}\right)+M_{+L}^{\mu}+l_{+L}^{\mu}+c_{+L}^{\mu}=j_{V^{+} L}^{\mu}
$$


Sector T:

$$
\begin{aligned}
& F_{+}^{\nu \mu}=\bar{c}_{1} V^{\nu \mu-}\left(V^{+} \text {-antisymmetric granular }\right) \\
& z_{+}^{[\nu \mu]}=\bar{c}_{2}\left({ }^{[-1]} z^{[\nu \mu]}+{ }^{[-2]}{ }^{[\nu \mu]}\right)\left(V^{+} \text {-antisymmetric collective }\right)
\end{aligned}
$$

Sector-L:

$$
\begin{aligned}
& S_{\alpha+}^{\alpha}=\bar{c}_{3} S_{\alpha}^{\alpha-},\left(V^{+} \text {-symmetric granular }\right) \\
& z_{\alpha+}^{\alpha}=\bar{c}_{4}\left(\stackrel{(-1)}{z}_{\alpha}+\stackrel{(-2)}{z}_{\alpha}^{\alpha}\right)+\bar{c}_{5}\left(\stackrel{(-1)}{\omega}_{\alpha}^{\alpha}+\stackrel{(-2)}{\omega}_{\alpha}^{\alpha}\right)\left(V^{+} \text {-symmetric collective }\right)
\end{aligned}
$$

Sector Mass:

$$
M_{+}^{\mu}=\mu_{V}^{2} V^{\mu-}
$$

Sector-London:

$$
l_{+}^{\mu}=\left(\Sigma_{\alpha}^{\mu}\right)_{\beta \nu} g^{\alpha \beta}\left(h_{11}^{-}\left(\stackrel{(11)}{z} \rho_{\rho}+\stackrel{(22)}{z} \rho_{\rho}+2{\stackrel{(11)}{\omega}{ }_{\rho}}_{\rho}+2 \stackrel{(22)}{\omega} \rho_{\rho}\right)\right) V^{-\nu}
$$

Sector-conglomerate:

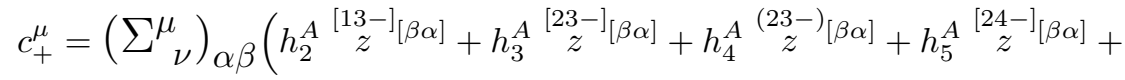

$$
\begin{aligned}
& \left.+h_{9}^{A} \stackrel{(24-)}{z}[\alpha \beta]+h_{10}^{A} \stackrel{(23-)}{\omega}[\alpha \beta]+h_{11}^{A} \stackrel{(24-)}{\omega}[\alpha \beta]\right) A^{\nu}+ \\
& +\left(\sum^{\mu}{ }_{\nu}\right)_{\alpha \beta}\left(h_{2}^{A} \stackrel{[13-]}{z}(\beta \alpha)+h_{7}^{A} \stackrel{[23-]_{(\beta \alpha)}}{ }+h_{8}^{A} \stackrel{(13-)}{z}(\beta \alpha)+h_{4}^{* A} \stackrel{(23-)}{z}(\beta \alpha)+\right. \\
& +h_{9}^{A} \stackrel{(24-)}{z}(\alpha \beta)+h_{10}^{A} \stackrel{(23-)}{\omega}(\alpha \beta)+h_{11}^{A} \stackrel{(24-)}{\omega}^{(\alpha \beta)}+ \\
& \left.+g^{\beta \alpha}\left(-h_{2}^{A} \stackrel{[13-]}{z}{ }_{\rho}-h_{2}^{A} \stackrel{[23-]}{z}{ }_{\rho}+h_{8}^{A} \stackrel{(23-)}{z}{ }_{\rho}+h_{13}^{A} \stackrel{(13-)}{\omega} \underset{\rho}{\rho}+h_{13}^{A} \stackrel{(23-)}{\omega} \underset{\rho}{\rho}\right)\right) A^{\nu}+ \\
& +\left(\Sigma^{\mu}{ }_{\nu}\right)_{\alpha \beta}\left(h_{2}^{U} \stackrel{[13-]}{z}[\alpha \beta]+h_{3}^{U} \stackrel{[14-]}{z}[\alpha \beta]+h_{4}^{U} \stackrel{[23-]}{z}[\beta \alpha]+h_{6}^{U} \stackrel{(13-)}{z}[\alpha \beta]+h_{7}^{U} \stackrel{(14-)}{z}[\alpha \beta]+\right. \\
& \left.+h_{8}^{U} \stackrel{(23-)}{z}[\alpha \beta]+h_{9}^{U} \stackrel{(13-)}{\omega}[\alpha \beta]+h_{10}^{U} \stackrel{(14-)}{\omega}[\alpha \beta]\right) U^{\nu} \\
& +\quad+\left(\Sigma^{\mu}{ }_{\alpha}\right)_{\beta \nu}\left(h_{2}^{U} \stackrel{[13-]}{z}(\alpha \beta)+h_{3}^{U} \stackrel{[14-]}{z}(\alpha \beta)+h_{4}^{U} \stackrel{[23-]}{z}(\alpha \beta)+h_{6}^{U} \stackrel{(13-)}{z}(\alpha \beta)+h_{7}^{U} \stackrel{(14-)}{z}(\alpha \beta)+\right. \\
& +h_{8}^{U} \stackrel{(23-)}{z}(\alpha \beta)+h_{9}^{U} \stackrel{(13-)}{\omega}{ }^{(\alpha \beta)}+h_{10}^{U} \stackrel{(14-)}{\omega}(\alpha \beta)^{(\alpha \beta}\left(h_{12}^{U} \stackrel{[13-]}{z} \underset{\rho}{\rho}-h_{4}^{U} \stackrel{[23-]}{z} \underset{\rho}{\rho}+\right. \\
& \left.\left.+h_{8}^{U} \stackrel{(13-)}{z} \underset{\rho}{\rho}+h_{8}^{U} \stackrel{(23-)}{z} \underset{\rho}{\rho}+h_{12}^{U} \stackrel{(13-)}{\omega} \stackrel{\rho}{\rho}+h_{12}^{U} \stackrel{(23-)}{\omega} \underset{\rho}{\rho}\right)\right) U^{\nu}+ \\
& +\left(\Sigma^{\mu}{ }_{\nu}\right)_{\alpha \beta}\left(h_{3}^{-}{ }^{[12]}[\beta \alpha]\right) V^{-\nu}+
\end{aligned}
$$

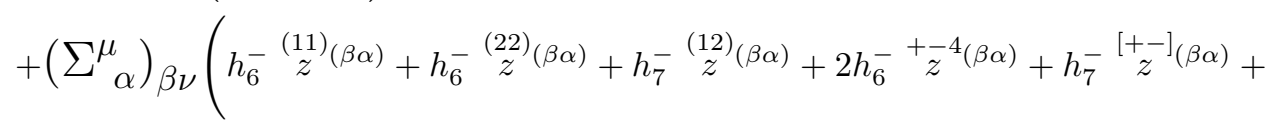

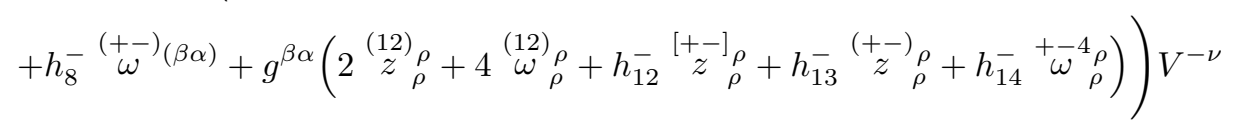


Sector current:

$$
\begin{aligned}
& j_{V^{+}}^{\mu}=\left(\sum^{\mu}{ }_{\nu}\right)_{\alpha \beta}\left(h_{1}^{A} V^{\beta \alpha-}\right) A^{\nu}+\left(\Sigma^{\mu}{ }_{\alpha}\right)_{\beta \nu}\left(h_{6}^{A} S^{\beta \alpha-}+g^{\beta \alpha}\left(h_{12}^{A} S_{\rho}^{\rho-}\right)\right)+ \\
& +\left(\Sigma^{\mu}{ }_{\nu}\right)_{\alpha \beta}\left(h_{1}^{U} V^{\beta \alpha-}\right) U^{\nu}+\left(\Sigma^{\mu}{ }_{\alpha}\right)_{\beta \nu}\left(h_{5}^{U} S^{\beta \alpha-}+g^{\beta \alpha}\left(h_{11}^{U} S_{\rho}^{\rho-}\right)\right)+ \\
& +\left(\Sigma^{\mu}{ }_{\nu}\right)_{\alpha \beta}\left(h_{1}^{-} F^{\beta \alpha}+h_{2}^{-} U^{\beta \alpha}\right) V^{\nu-}+ \\
& +\left(\Sigma^{\mu}{ }_{\alpha}\right)_{\beta \nu}\left(h_{4}^{-} S^{\beta \alpha 1}+h_{5}^{-} S^{\beta \alpha 2}+g^{\beta \alpha}\left(h_{9}^{-} S_{\rho}^{\rho 1}+h_{10}^{-} S_{\rho}^{\rho 2}\right)\right) V^{\beta \alpha-}+
\end{aligned}
$$

The corresponding continuity equation is

$$
\square\left(S_{\alpha+}^{\alpha}+z_{\alpha+}^{\alpha}\right)=\partial \cdot\left(j_{+}+l_{+}+c_{+}+M_{+}\right)
$$

where the parameters $\bar{c}_{1}, \ldots$ are defined in Appendix A.

The corresponding electromagnetic waves expressions are:

$V_{\mu}^{+}$-spin-1:

$$
\square\left(F_{\nu \mu}^{+}+z_{[\nu \mu]}^{+}\right)=\partial_{\mu} d_{\nu}^{+}-\partial_{\nu} d_{\mu}^{+}+\partial_{\mu} M_{\nu}^{+}-\partial_{\nu} M_{\mu}^{+}+\partial^{\alpha} f_{\alpha \mu \nu}^{+}
$$

where

$$
d_{\mu}^{+}=l_{\mu}^{+T}+c_{\mu}^{+}+j_{\mu V^{+}}^{T} \quad \text { e } \quad f_{\alpha \mu \nu}^{+}=\bar{c}_{2}\left(f_{\alpha \mu \nu}^{[-1]}+f_{\alpha \mu \nu}^{[-2]}\right)
$$

$V_{\mu}^{+}$-spin-0:

$$
\square\left(S_{\alpha+}^{\alpha}+z_{\alpha+}^{\alpha}\right)=\partial \cdot\left(j_{+}-l_{+}-c_{+}\right)
$$

For negative charge field, $V_{\mu}^{-}$(Negative Massive Photon), one gets

$V_{\mu}^{-}$-spin-1:

$$
\partial_{\nu}\left(F_{-}^{\nu \mu}+z_{-}^{[\nu \mu]}\right)+M_{-T}^{\mu}+c_{-T}^{\mu}+l_{-T}^{\mu}=j_{V^{-T}}^{\mu}
$$

$V_{\mu}^{-}$-spin-0:

$$
\partial^{\mu}\left(S_{\alpha-}^{\alpha}+z_{\alpha-}^{\alpha}\right)+M_{-L}^{\mu}+c_{-L}^{\mu}+l_{-L}^{\mu}=j_{V^{-} L}^{\mu}
$$


Sector T:

$$
\begin{aligned}
& F_{-}^{\nu \mu}=\bar{c}_{1} V^{\nu \mu+},\left(V^{-} \text {-antisymmetric granular }\right) \\
& z_{-}^{[\nu \mu]}=\bar{c}_{2}\left(\stackrel{[+1]}{z}[\nu \mu]+{ }_{z}^{[+2]}[\nu \mu]\right)\left(V^{-} \text {-antisymmetric collective }\right)
\end{aligned}
$$

Sector L:

$$
\begin{aligned}
& S_{\alpha-}^{\alpha}=\bar{c}_{3} S_{\alpha}^{\alpha+},\left(V^{-} \text {-symmetric granular }\right) \\
& z_{\alpha-}^{\alpha}=\bar{c}_{4}\left(\stackrel{(+1)}{z}_{\alpha}^{\alpha}+\stackrel{(+2)}{z}_{\alpha} \alpha\right)+\bar{c}_{5}\left(\stackrel{(+1)}{\omega}_{\alpha}^{\alpha}+\stackrel{(+2)}{\omega}_{\alpha}^{\alpha}\right)\left(V^{-} \text {-symmetric collective }\right)
\end{aligned}
$$

Sector Mass:

$$
M_{-}^{\mu}=\mu_{V}^{2} V^{\mu-}
$$

Sector-London:

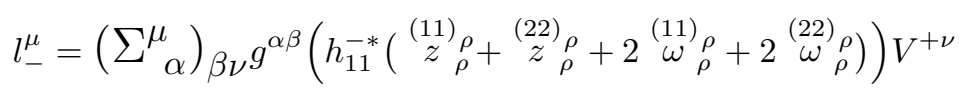

Sector-conglomerate:

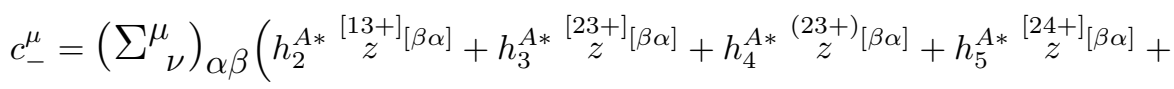

$$
\begin{aligned}
& \left.+h_{9}^{A *}{ }_{z}^{(24+)}[\alpha \beta]+h_{10}^{A *} \stackrel{(23+)}{\omega}[\alpha \beta]+h_{11}^{A *} \stackrel{(24+)}{\omega}[\alpha \beta]\right) A^{\nu}+ \\
& +\left(\sum^{\mu}{ }_{\nu}\right)_{\alpha \beta}\left(h_{2}^{A *}{ }_{z}^{[13+]}(\beta \alpha)+h_{7}^{A *} \underset{z}{[23+]}(\beta \alpha)+h_{8}^{A *} \underset{z}{(13+)}(\beta \alpha)+h_{4}^{A} \underset{z}{(23+)}(\beta \alpha)+\right. \\
& +h_{9}^{A *} \underset{z}{(24+)}(\alpha \beta)+h_{10}^{A *} \stackrel{(23+)}{\omega}(\alpha \beta)+h_{11}^{A *} \stackrel{(24+)}{\omega}(\alpha \beta)+ \\
& \left.+g^{\beta \alpha}\left(-h_{2}^{A *}{ }_{z}^{[13+]} \rho-h_{2}^{A *}{ }_{z}^{[23+]} \rho+h_{8}^{A *} \underset{z}{\rho}{ }_{\rho}^{(23+)}+h_{13}^{A *} \underset{\rho}{\omega}+h_{13}^{A *} \stackrel{(23+)}{\omega} \rho\right)\right) A^{\nu}+
\end{aligned}
$$

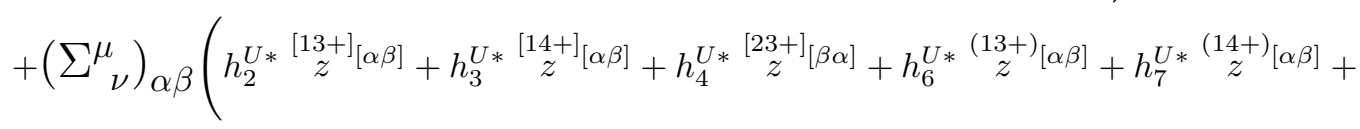

$$
\begin{aligned}
& \left.+h_{8}^{U *} \stackrel{(23+)}{z}[\alpha \beta]+h_{9}^{U *} \stackrel{(13+)}{\omega}[\alpha \beta]+h_{10}^{U *} \stackrel{(14+)}{\omega}[\alpha \beta]\right) U^{\nu}
\end{aligned}
$$

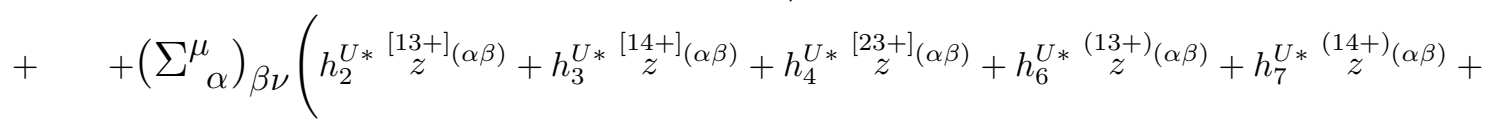

$$
\begin{aligned}
& +h_{8}^{U *}{ }_{z}^{(23+)}(\alpha \beta)+h_{9}^{U *} \stackrel{(13+)}{\omega}(\alpha \beta)+h_{10}^{U *} \stackrel{(14+)}{\omega}(\alpha \beta)+g^{\alpha \beta}\left(h_{12}^{U *}{ }_{z}^{[13+]}{ }_{\rho}-h_{4}^{U *}{ }_{z}^{[23+]} \rho+\right. \\
& \left.\left.+h_{8}^{U *} \stackrel{(13+)}{z} \underset{\rho}{\rho}+h_{8}^{U *} \stackrel{(23+)}{z} \underset{\rho}{\rho}+h_{12}^{U *} \stackrel{(13+)}{\omega} \underset{\rho}{\rho}+h_{12}^{U *} \stackrel{(23+)}{\omega} \underset{\rho}{\rho}\right)\right) U^{\nu}+
\end{aligned}
$$

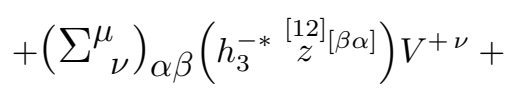

$$
\begin{aligned}
& +\left(\Sigma^{\mu}{ }_{\alpha}\right)_{\beta \nu}\left(h_{6}^{-*}{\underset{z}{(11)}(\beta \alpha)}^{(-*} h_{6}^{-(22)}(\beta \alpha)+h_{7}^{-*}{ }_{z}^{(12)}(\beta \alpha)+2 h_{6}^{-*}+{ }_{z}^{-4}(\beta \alpha)+h_{7}^{-*}{ }_{z}^{[+-]}(\beta \alpha)+\right.
\end{aligned}
$$

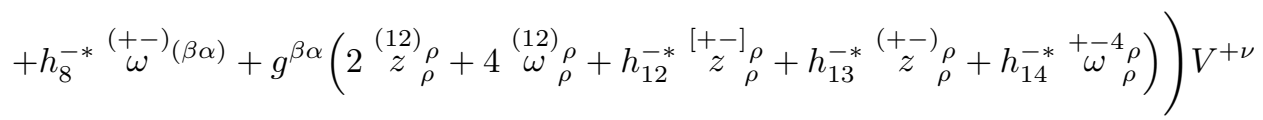


Sector current:

$$
\begin{aligned}
& j_{V^{-}}^{\mu}=\left(\Sigma^{\mu}{ }_{\nu}\right)_{\alpha \beta}\left(h_{1}^{A *} V^{\beta \alpha+}\right) A^{\nu}+\left(\Sigma^{\mu}{ }_{\alpha}\right)_{\beta \nu}\left(h_{6}^{A *} S^{\beta \alpha+}+g^{\beta \alpha}\left(h_{12}^{A *} S_{\rho}^{\rho-}\right)\right)+ \\
& +\left(\Sigma^{\mu}{ }_{\nu}\right)_{\alpha \beta}\left(h_{1}^{U *} V^{\beta \alpha+}\right) U^{\nu}+\left(\Sigma^{\mu}{ }_{\alpha}\right)_{\beta \nu}\left(h_{5}^{U *} S^{\beta \alpha+}+g^{\beta \alpha}\left(h_{11}^{U *} S_{\rho}^{\rho+}\right)\right)+ \\
& +\left(\Sigma^{\mu}{ }_{\nu}\right)_{\alpha \beta}\left(h_{1}^{-*} F^{\beta \alpha}+h_{2}^{-*} U^{\beta \alpha}\right) V^{\nu+}+ \\
& +\left(\Sigma^{\mu}{ }_{\alpha}\right)_{\beta \nu}\left(h_{4}^{-*} S^{\beta \alpha 1}+h_{5}^{-*} S^{\beta \alpha 2}+g^{\beta \alpha}\left(h_{9}^{-*} S_{\rho}^{\rho 1}+h_{10}^{-*} S_{\rho}^{\rho 2}\right)\right) V^{\beta \alpha-}+
\end{aligned}
$$

The corresponding continuity equation is

$$
\square\left(S_{\alpha-}^{\alpha}+z_{\alpha-}^{\alpha}\right)=\partial \cdot\left(j_{-}+l_{-}+M_{-}+c_{-}\right)
$$

The corresponding electromagnetic expressions are :

$V_{\mu}^{-}$-spin-1:

$$
\square\left(F_{\nu \mu}^{-}+z_{[\nu \mu]}^{-}\right)=\partial_{\mu} d_{\nu}^{-}-\partial_{\nu} d_{\mu}^{-}+\partial_{\mu} M_{\nu}^{-}-\partial_{\nu} M_{\mu}^{-}+\partial^{\alpha} f_{\alpha \mu \nu}^{-}
$$

where

$$
d_{\mu}^{-}=l_{\mu}^{-T}+c_{\mu}^{-}+j_{\mu V^{-}}^{T} \quad \text { e } \quad f_{\alpha \mu \nu}^{-}=\bar{c}_{2}^{*}\left(f_{\alpha \mu \nu}^{[+1]}+f_{\alpha \mu \nu}^{[+2]}\right)
$$

$V_{\mu}^{-}$-spin-0:

$$
\square\left(S_{\alpha-}^{\alpha}+z_{\alpha-}^{\alpha}\right)=\partial \cdot\left(j_{-}-l_{-}-c_{-}\right)
$$

\section{Noether's Theorem}

The model proposes the symmetry $U(1) \times S O(2)_{\text {global }}$ invariance under a common gauge parameter. It contains the global gauge invariance of first and second species. It yields the following three Noether identities [28]

$$
\begin{array}{cr}
\partial_{\mu} J_{N}^{\mu}=0 \quad \text { (electric charge conservation) } \\
\partial_{\mu} K^{\mu \nu}+J_{N}^{\nu}=0 \quad \text { (symmetry equation) } \\
K^{\mu \nu} \partial_{\mu} \partial_{\nu} \alpha=0 \quad \text { (symmetry constraint). }
\end{array}
$$

The first one means the total electric charge conservation is a consequence from the first specie gauge invariance. Any Lagrangian which respects electric charge conservation will automatically be invariant be invariant under the first kind transformation. Thus considering that the photon and massive photon carry no charge, they will be invariant under the transformations $A_{\mu} \rightarrow A_{\mu}$ and $U_{\mu} \rightarrow U_{\mu}$, while the charged photons will transform as $V_{\mu}^{ \pm} \rightarrow e^{ \pm i q \alpha} V_{\mu}^{ \pm}$. It gives, 


$$
\begin{aligned}
& J_{N}^{\mu} \equiv i q\left(V_{\nu}^{+} \frac{\partial L}{\partial\left(\partial_{\mu} V_{\nu}^{+}\right)}-V_{\nu}^{-} \frac{\partial L}{\partial\left(\partial_{\mu} V_{\nu}^{-}\right)}\right)
\end{aligned}
$$

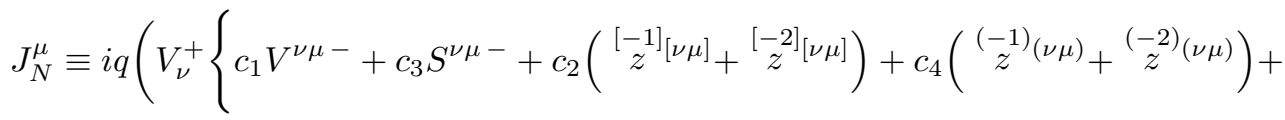

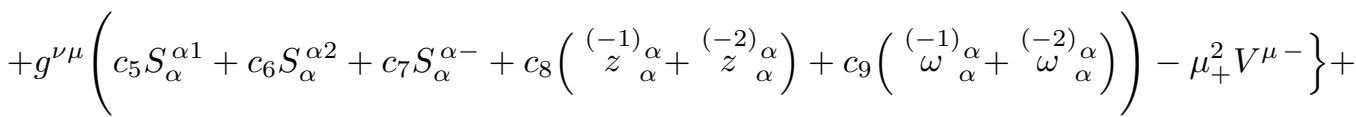

$$
\begin{aligned}
& -V_{\nu}^{-}\left\{d_{1} V^{\nu \mu+}+d_{3} S^{\nu \mu+}+d_{2}\left(\underset{z}{[+1]}[\nu \mu]+{ }_{z}^{[+2]}[\nu \mu]\right)+d_{4}(\underset{z}{(+1)}(\nu \mu)+\stackrel{(+2)}{z}(\nu \mu))+\right. \\
& \left.+g^{\nu \mu}\left(d_{5} S_{\alpha}^{\alpha 1}+d_{6} S_{\alpha}^{\alpha 2}+d_{7} S_{\alpha}^{\alpha+}+d_{8}\left(\stackrel{(+1)}{z}_{\alpha}+\stackrel{(+2)}{z}_{\alpha}\right)+d_{9}\left(\stackrel{(+1)}{\omega}_{\alpha}^{\alpha}+\stackrel{(+2)}{\omega}_{\alpha}^{\alpha}\right)\right)+\mu_{+}^{2} V^{\mu+}\right\}
\end{aligned}
$$

\section{Conclusion}

Electromagnetism is the theory of electric charge and spin. Nevertheless spin is not a well understood physical attribute. Its heuristic presence is given by the Pauli term $\vec{\mu} \cdot \vec{B}$ where $\vec{\mu}=\frac{e \hbar \vec{S}}{2 m}$. Consequently, physics has to investigate on $\vec{S}$ origin. Although classic and quantum mechanics provide own arguments it is necessary to be considered under field theory. Given a field relate its spin physics. Explicit the $\vec{S}$ vector at equations of motion.

The field theory performance is to systematize the quantum numbers inserted in a field. Mass is obtained as the pole of a two point Green's function, charges from conservation laws, discrete symmetries directly from fields properties. However the field-spin physics is not clear. Although the Lorentz group associate quantum numbers to spins the equations of motion do not show their presence. Maxwell and QED propagates spin-1 but does not show its interactions.

Thus, despite all heuristic and technological developments, spin is still a not well understood physical entity. The difficulty is that QED does not contain a term $F_{\mu \nu} A^{\nu}$ as a source. Consequently, it is unable to provide a term like $\vec{\mu}_{A} \cdot \vec{B}$ from an initial Lagrangian. Something is missing on Maxwell electromagnetism. It provides the photon degrees of freedom but does not explicit on its spin contents. Eq.(2.1) is introduced by hand.

The argument here is that spin is an intrinsic entity compatible with relativity and group theory. Based on that, one should derive spin as a vector with angular momentum properties. Experimentally, we know whether a particle has spin $\vec{S}$ it will interact with magnetic field as $\vec{B} \cdot \vec{S}$. Theoretically, the challenge is to find out a Lagrangian able to derive $\vec{\mu} \cdot \vec{B}$ from equations of motion.

Our effort here is to introduce spin at ab initio. Be created before other physical entities as fields strenghts. For this, one introduces Lorentz Group Lie Algebra valued fields. At this way one associates fields and group theory through eq.(2.5). And so, eq.(3.1) becames a new origin to express an electromagnetism with electric charge and spin together.

Spintronics has been quite developed for spin- $\frac{1}{2}$. The novelty is that the vector bosons quadruplet opens a new working hypothesis for vector spintronics. Proposes an electromagnetism based on vectors bosons spin currents. Understand that physics laws are challenged regarding on the understanding of spin currents [29]. 
Eq. (2.5) understands that the spin physical picture should be originated from fields associated to Lorentz group. Taking this definition to the nonlinear Four Bosons Electromagnetism, it yields the eq.(3.56) spin-valued Lagrangian. Then, in order to identify the spin interaction we are going to reduce the Lagrangian comprehension. Rewriting eq.(3.7) in spin terms, one gets the minimal coupling between fields as

$$
\mathcal{L}^{\text {spin }} \approx g_{I J} F_{\alpha \beta} A_{I}^{\mu}\left(\Sigma^{\alpha \beta}\right)_{\mu \nu} A_{J}^{\nu}
$$

where eq.(7.1) introduces a constant $g_{I J}$ which is not necessary the electric charge, $F_{\alpha \beta}$ means the EM field, the spin density $A_{I}^{\mu}\left(\Sigma^{\alpha \beta}\right)_{\mu \nu} A_{J}^{\nu}$, and $\Sigma_{\mu \nu}^{\alpha \beta} A_{I}^{\nu}$ the spin valued field.

Thus in order to identify the spin interaction we are going to focus the generic eq.(4.1) into

$$
\partial_{\nu} F_{I}^{\nu \mu}=g F_{\alpha \beta}\left(\Sigma^{\alpha \beta}\right)_{\mu}^{\nu} A_{I}^{\mu}+\text { other terms }
$$

Eq.(7.2) shows the spin matrix acting on the field $A_{I}^{\mu}$. It introduces the coupling between an external photonic EM field $F_{\alpha \beta}$ with the spin of a generic field $A_{I}^{\mu}$ as $\left(\Sigma^{\alpha \beta}\right)_{\mu}^{\nu} A_{I}^{\mu}$. Expanding

$$
F_{\alpha \beta}\left(\Sigma^{\alpha \beta}\right)_{\nu}^{\mu} A_{I}^{\nu}=F_{0 i}\left(\Sigma^{0 i}\right)_{\nu}^{\mu} A^{\nu}+F_{i j}\left(\Sigma^{i j}\right)_{\nu}^{\mu} A_{I}^{\nu}
$$

and considering no presence of electric field, it gives

$$
F_{\alpha \beta}\left(\Sigma^{\alpha \beta}\right)_{\nu}^{\mu} A_{I}^{\nu}=-\left(\epsilon_{i j k} B_{k}\right)\left(\Sigma^{i j}\right)_{\nu}^{\mu} A_{I}^{\nu}
$$

Defining as the spin vector field

$$
S_{k} \equiv \epsilon_{i j k} \Sigma_{j k}
$$

eq.(7.5) rewrites the equation of motion as

$$
g F_{\alpha \beta}\left(\Sigma^{\alpha \beta}\right)_{\nu}^{\mu} A_{I}^{\nu}=-g\left(B_{k} S_{k}\right)_{\nu}^{\mu} A_{I}^{\nu}=-g(\vec{B} \cdot \vec{S})_{\nu}^{\mu} A_{I}^{\nu}
$$

Thus the quadri-magnetic moment of a given field $A_{I}^{\mu}$ is expressed as

$$
\vec{\mu}_{I}^{\mu}=\vec{S}_{\nu}^{\mu} A_{I}^{\nu}
$$

A covariant magnetic moment is obtained. Eq.(7.7) determines the spin as a vector entity as expected from the Pauli relationship $\vec{\mu} \cdot \vec{B}$. It provides two extensions. It contains an index $\mu$ which is related to the space-time equations of motion. It couples with scalar and vector fields. 
It yields,

$$
\vec{\mu}_{I}^{\mu}=\vec{S}_{0}^{\mu} A_{I}^{0}+\vec{S}_{i}^{\mu} A_{I}^{i}
$$

Notice that the spin introduces the meaning of potential fields as considered by the Ahranov-Bohm effect [30].Including the electric field at eq.(7.2) one gets the Ahanov-Casher term $\vec{\mu} \times \vec{E}$. Another interpretation for eq.(7.6) is to consider the magnetic moment as a tensor $F_{\alpha \beta}\left(\Sigma^{\alpha \beta}\right)_{\mu \nu} A_{I}^{\nu}=\vec{B} \cdot \vec{\mu}_{\mu \nu}$.

For the vector field case, one gets for eq.(7.7)

$$
\vec{\mu}_{z, s=1}^{i}=g \frac{e \hbar}{2 m c} \vec{S}
$$

where $\left(S_{i}\right)_{j k}=-i \epsilon_{i j k}$ are the matrix elements corresponding to $\mathrm{SU}(2)$ adjoint representations $(s=1,2 s+1=3)$. It gives,

$$
S_{1}=\left(\begin{array}{ccc}
0 & 0 & 0 \\
0 & 0 & -i \\
0 & -i & 0
\end{array}\right), S_{2}=\left(\begin{array}{ccc}
0 & 0 & i \\
0 & 0 & 0 \\
i & 0 & 0
\end{array}\right), S_{3}=\left(\begin{array}{ccc}
0 & -i & 0 \\
i & 0 & 0 \\
0 & 0 & 0
\end{array}\right)
$$

As a conclusion, we notice that three aspects are derived by taking fields Lorentz valued as ab initio. Eq.(2.5) prescribes the spin as a physical entity preceding the EM fields, eq.(7.5) discovers the spin vectorial nature and the Pauli term is rewritten by eq.(7.6). This conciseness introduces Lagrangian (3.56) as candidate for describing spintronics-1 [31].

\section{Appendix A. Collective Fields}

Alongside the usual granular fields, new collective fields appear, defined by:

$$
\begin{aligned}
& z_{\mu \nu}=\gamma_{I J} G_{\mu}^{I} G_{\nu}^{J} \\
& =\gamma_{11} G_{\mu}^{1} G_{\nu}^{1}+\gamma_{12} G_{\mu}^{1} G_{\nu}^{2}+\gamma_{13} G_{\mu}^{1} G_{\nu}^{3}+\gamma_{14} G_{\mu}^{1} G_{\nu}^{4}+ \\
& +\gamma_{21} G_{\mu}^{2} G_{\nu}^{1}+\gamma_{22} G_{\mu}^{2} G_{\nu}^{2}+\gamma_{23} G_{\mu}^{2} G_{\nu}^{3}+\gamma_{24} G_{\mu}^{2} G_{\nu}^{4}+ \\
& +\gamma_{31} G_{\mu}^{3} G_{\nu}^{1}+\gamma_{32} G_{\mu}^{3} G_{\nu}^{2}+\gamma_{33} G_{\mu}^{3} G_{\nu}^{3}+\gamma_{34} G_{\mu}^{3} G_{\nu}^{4}+ \\
& +\gamma_{41} G_{\mu}^{4} G_{\nu}^{1}+\gamma_{42} G_{\mu}^{4} G_{\nu}^{2}+\gamma_{43} G_{\mu}^{4} G_{\nu}^{3}+\gamma_{44} G_{\mu}^{4} G_{\nu}^{4}
\end{aligned}
$$

The collective antisymmetric fields are

$$
z_{[\mu \nu]}=\left(\begin{array}{cccc}
0 & z^{[1,2]} & z^{[1,+]} & z^{[1,-]} \\
-z^{[1,2]} & 0 & z^{[2,+]} & z^{[2,-]} \\
-z^{[+, 1]} & -z^{[+, 2]} & 0 & z^{[+,-]} \\
-z^{[-, 1]} & -z^{[-, 2]} & -z^{[-,+]} & 0
\end{array}\right)
$$


there are also 10 symmetric collective fields,

$$
z_{(\mu \nu)}=\left(\begin{array}{llll}
z^{(1,1)} & z^{(1,2)} & z^{(1,+)} & z^{(1,-)} \\
z^{(1,2)} & z^{(2,2)} & z^{(2,+)} & z^{(2,-)} \\
z^{(1,+)} & z^{(2,+)} & z^{(+,+)} & z^{(+,-)} \\
z^{(1,-)} & z^{(2,-)} & z^{(+,-)} & z^{(-,-)}
\end{array}\right)
$$

Considering the fields set $\left\{A_{\mu}, U \mu, V_{\mu}^{ \pm}\right\}$one gets the following group of collective fields,

$$
\begin{aligned}
& \stackrel{(11)}{z} \mu \nu \equiv \gamma_{(11)} A^{\mu} A^{\nu}, \quad{ }_{z}^{(22)} \mu \nu \equiv \gamma_{(22)} U^{\mu} U^{\nu}, \quad \underset{z}{(12)} \mu \nu \equiv \gamma_{(12)} A^{\mu} U^{\nu} \\
& { }_{z}^{(21)} \mu \nu \equiv \gamma_{(21)} U^{\mu} A^{\nu} \quad \stackrel{(11)}{z}_{\mu}=\gamma_{(11)} A_{\mu} A^{\mu}, \quad \stackrel{(22)}{z}_{\mu}^{\mu}=\gamma_{(22)} U_{\mu} U^{\mu} \text {, } \\
& \stackrel{(12)}{{ }^{\prime}} \underset{\mu}{\mu}=\gamma_{(12)} A_{\mu} U^{\mu}, \quad{ }_{z}^{[12]} \mu \nu \equiv \gamma_{[12]} A^{\mu} U^{\nu}, \quad{ }_{z}^{[21]} \mu \nu \equiv \gamma_{[21]} U^{\mu} A^{\nu} \text {, } \\
& { }_{z}^{(13+)} \mu \nu \equiv \gamma_{(13)} A^{\mu} V^{\nu+}, \quad{ }^{(13-)} \mu \nu \equiv\left\{\begin{array}{c}
(13+) \\
z
\end{array}\right\}^{*}=\gamma_{(13)} A^{\mu} V^{\nu-} \text {, } \\
& \stackrel{(14+)}{z} \mu \nu \equiv \gamma_{(14)} A^{\mu} V^{\nu+}, \quad \stackrel{(14-)}{z} \mu \nu \equiv\{\stackrel{(14+)}{z} \mu \nu\}^{*}=\gamma_{(14)} A^{\mu} V^{\nu-} \text {, }
\end{aligned}
$$

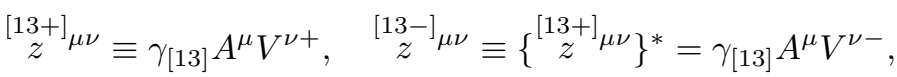

$$
\begin{aligned}
& { }^{[14+]} \mu \nu \equiv \gamma_{[14]} A^{\mu} V^{\nu+}, \quad{ }_{z}^{[14-]} \mu \nu \equiv\left\{{ }^{[14+]} \mu \nu\right\}^{*}=\gamma_{[14]} A^{\mu} V^{\nu-} \text {, } \\
& \stackrel{(23+)}{z} \mu \nu \equiv \gamma_{(23)} U^{\mu} V^{\nu+}, \quad \stackrel{(23-)}{z} \mu \nu \equiv\{\stackrel{(23+)}{z} \mu \nu\}^{*}=\gamma_{(23)} U^{\mu} V^{\nu-} \text {, } \\
& \stackrel{(24+)}{z} \mu \nu \equiv \gamma_{(24)} U^{\mu} V^{\nu+}, \quad \underset{z}{(24-)} \mu \nu \equiv\{\underset{z}{(24+)} \mu \nu\}^{*}=\gamma_{(24)} U^{\mu} V^{\nu-} \text {, } \\
& { }_{z}^{[23+]} \mu \nu \equiv \gamma_{[23]} U^{\mu} V^{\nu+}, \quad{ }_{z}^{[23-]} \mu \nu \equiv\left\{{ }^{[23+]} \mu \nu\right\}^{*}=\gamma_{[23]} U^{\mu} V^{\nu-} \text {, } \\
& { }_{z}^{[24+]} \mu \nu \equiv \gamma_{[24]} U^{\mu} V^{\nu+}, \quad{ }_{z}^{[24-]} \mu \nu \equiv\left\{{ }_{z}^{[24+]} \mu \nu\right\}^{*}=\gamma_{[24]} U^{\mu} V^{\nu-} \text {, } \\
& { }^{+-3} \mu \nu \equiv \gamma_{(33)} V^{\mu+} V^{\nu-}, \quad{ }_{z}^{+3} \mu \nu \equiv\left\{{ }^{+-3} \mu \nu\right\}^{*}=\gamma_{(33)} V^{\mu-} V^{\nu+} \text {, } \\
& { }^{+-4} \mu \nu \equiv \gamma_{(44)} V^{\mu+} V^{\nu-}, \quad{ }_{z}^{+4} \mu \nu \equiv\left\{{ }^{+-4} \mu \nu\right\}^{*}=\gamma_{(44)} V^{\mu-} V^{\nu+} \text {, } \\
& { }_{z}^{(+-)} \mu \nu \equiv-i \gamma_{(34)} V^{\mu+} V^{\nu-}, \quad{ }_{z}^{(-+)} \mu \nu \equiv\left\{{ }^{(+-)} \mu \nu\right\}^{*}=i \gamma_{(34)} V^{\mu-} V^{\nu+} \text {, } \\
& { }_{z}^{[+-]} \mu \nu \equiv-i \gamma_{[34]} V^{\mu+} V^{\nu-}, \quad{ }_{z}^{[-+]} \mu \nu \equiv\left\{{ }^{[+-]} \mu \nu\right\}^{*}=i \gamma_{[34]} V^{\mu-} V^{\nu+}, \\
& \stackrel{(+1)}{z} \mu \nu \equiv\left(\gamma_{(13)}+i \gamma_{(14)}\right) A^{\mu} V^{\nu+}, \quad \stackrel{(-1)}{z} \mu \nu \equiv\{\stackrel{(+1)}{z} \mu \nu\}^{*}, \\
& \stackrel{(+2)}{z} \mu \nu \equiv\left(\gamma_{(23)}+i \gamma_{(24)}\right) U^{\mu} V^{\nu+}, \quad \underset{z}{(-2)} \mu \nu \equiv\{\stackrel{(+2)}{z} \mu \nu\}^{*}, \\
& { }_{z}^{[+1]} \mu \nu \equiv\left(\gamma_{[13]}+i \gamma_{[14]}\right) A^{\mu} V^{\nu+}, \quad{ }_{z}^{[-1]} \mu \nu \equiv\left\{{ }_{z}^{[+1]} \mu \nu\right\}^{*} \text {, } \\
& { }_{z}^{[+2]} \mu \nu \equiv\left(\gamma_{[23]}+i \gamma_{[24]}\right) U^{\mu} V^{\nu+}, \quad{ }_{z}^{[-2]} \mu \nu \equiv\left\{{ }^{[+2]} \mu \nu\right\}^{*} \text {. }
\end{aligned}
$$

Similarly, one adds the $\omega$ collective fields associated to $\tau_{i j}$

$$
\begin{aligned}
\stackrel{(11)}{\omega}_{\alpha} & \equiv \tau_{(11)} A^{\alpha} A_{\alpha}, \quad \stackrel{(22)}{\omega}_{\alpha} \equiv \tau_{(22)} U^{\alpha} U_{\alpha}, \quad \stackrel{(12)}{\omega}_{\alpha} \equiv \tau_{(12)} A^{\alpha} U_{\alpha}, \\
{ }^{+-3}{ }_{\alpha} & \equiv \tau_{(33)} V^{\alpha+} V_{\alpha-}, \quad \stackrel{(+1)}{\omega}_{\alpha} \equiv\left(\tau_{(13)}+i \tau_{(14)}\right) A^{\alpha} V_{\alpha}^{+},
\end{aligned}
$$


Rewriting $z_{[\mu \nu]}$ and $z_{(\mu \nu)}$, one gets

$$
\begin{aligned}
& z_{[\mu \nu]}=\gamma_{[12]}\left(A_{\mu} U_{\nu}-A_{\nu} U_{\mu}\right)+\frac{1}{\sqrt{2}}\left(\gamma_{[13]}+i \gamma_{[14]}\right)\left(A_{\mu} V_{\nu}^{+}-A_{\nu} V_{\mu}^{+}\right)+ \\
& +\frac{1}{\sqrt{2}}\left(\gamma_{[13]}-i \gamma_{[14]}\right)\left(A_{\mu} V_{\nu}^{-}-A_{\nu} V_{\mu}^{-}\right)+\frac{1}{\sqrt{2}}\left(\gamma_{[23]}+i \gamma_{[24]}\right)\left(U_{\mu} V_{\nu}^{+}-U_{\nu} V_{\mu}^{+}\right)+ \\
& +\frac{1}{\sqrt{2}}\left(\gamma_{[23]}+i \gamma_{[24]}\right)\left(U_{\mu} V_{\nu}^{-}-U_{\nu} V_{\mu}^{-}\right)-\gamma_{[34]}\left(V_{\mu}^{+} V_{\nu}^{-}-V_{\mu}^{-} V_{\nu}^{+}\right)
\end{aligned}
$$

or

$$
z_{[\mu \nu]}=2 \stackrel{[12]}{z}_{[\mu \nu]}+\sqrt{2} \stackrel{[+1]}{z}_{[\mu \nu]}+\sqrt{2} \stackrel{[-1]}{z}_{[\mu \nu]}+2 \stackrel{[+2]}{z}[\mu \nu]_{[\mu}+\sqrt{2} \stackrel{[-2]}{z}_{[\mu \nu]}+{ }^{[+-]}{ }_{[\mu \nu]}^{[}
$$

And,

$$
\begin{aligned}
& z_{(\mu \nu)}=\gamma_{11} A_{\mu} A_{\nu}+\gamma_{22} U_{\mu} U_{\nu}+\frac{1}{2}\left(\gamma_{33}-\gamma_{44}\right)\left(V_{\mu}^{+} V_{\nu}^{-}+V_{\mu}^{-} V_{\nu}^{+}\right)+ \\
& +\frac{1}{2}\left(\gamma_{33}+\gamma_{44}\right)\left(V_{\mu}^{+} V_{\nu}^{-}+V_{\mu}^{-} V_{\nu}^{+}\right)+\gamma_{(12)}\left(A_{\mu} U_{\nu}+U_{\mu} A_{\nu}\right)+ \\
& +\frac{1}{\sqrt{2}}\left(\gamma_{(13)}+i \gamma_{(14)}\right)\left(A_{\mu} V_{\nu}^{+}+A_{\nu} V_{\mu}^{+}\right)+\frac{1}{\sqrt{2}}\left(\gamma_{(13)}-i \gamma_{(14)}\right)\left(A_{\mu} V_{\nu}^{-}+A_{\nu} V_{\mu}^{-}\right)+ \\
& +\frac{1}{\sqrt{2}}\left(\gamma_{(23)}+i \gamma_{(24)}\right)\left(U_{\mu} V_{\nu}^{+}+U_{\nu} V_{\mu}^{+}\right)+\frac{1}{\sqrt{2}}\left(\gamma_{(23)}-i \gamma_{(24)}\right)\left(U_{\mu} V_{\nu}^{-}+U_{\nu} V_{\mu}^{-}\right)+ \\
& +i \gamma_{(34)}\left(V_{\mu}^{+} V_{\nu}^{-}+V_{\mu}^{-} V_{\nu}^{+}\right)
\end{aligned}
$$

\section{Appendix B. Equations of motion free coefficients}

The equations of motion develop the following parameters written in terms of the original free Lagrangian coefficients, $\mathrm{eq}(2.2)$ :

B.1. Field $A_{\mu}$

\section{B.1.1. Left Side}

$$
\begin{aligned}
& \bar{a}_{1}=4\left(a_{1}+b_{(11)}\right), \quad \bar{a}_{2}=8 b_{1}, \quad \bar{a}_{3}=8 \beta_{1}, \\
& \bar{a}_{4}=4\left(b_{(11)}+c_{(11)}\right)+\xi_{(11)} \\
& \bar{a}_{5}=4 c_{(12)}+\xi_{(22)} \\
& \bar{a}_{6}=2\left(2 \rho_{1}-\beta_{1}\right), \quad \bar{a}_{7}=4\left(\beta_{1}+4 \rho_{1}\right)
\end{aligned}
$$


B.1.2. Field $A_{\mu}$ Right Side : Field $A_{\mu}$

$$
\begin{aligned}
& f_{1}^{A}=4 \gamma_{(11)} \beta_{2}-4 \beta_{1} \gamma_{(12)} \quad f_{2}^{A}=4 \gamma_{(11)} \quad f_{3}^{A}=2 f_{3}^{A}, \\
& f_{4}^{A}=8\left(\gamma_{(11)}+2 \tau_{(11)}\right), \quad f_{5}^{A}=2 f_{4}, \\
& f_{6}^{A}=2 \gamma_{(11)}\left(2 \rho_{1}-\beta_{1}\right)+8 \tau_{(11)}\left(\beta_{1}+4 \rho_{1}\right), \\
& f_{7}^{A}=4 \gamma_{(11)} \rho_{2}-2 \beta_{1} \gamma_{(12)}+8 \tau_{(11)}\left(\beta_{2}+4 \rho_{2}\right)
\end{aligned}
$$

B.1.3. Field $A_{\mu}$ Right Side : Field $U_{\mu}$

$$
\begin{aligned}
& f_{1}^{U}=-4 \gamma_{[12]} b_{1}, \quad f_{2}^{U}=-4 \gamma_{[12]} b_{1}, \quad f_{3}^{U}=-8 \gamma_{[12]}, \\
& f_{4}^{U}=16\left(\gamma_{(12)}+2 \tau_{(12)}\right), \quad f_{5}^{U}=-4 \gamma_{(12)} \beta_{2}-4 \beta_{1} \gamma_{(22)}, \\
& f_{6}^{U}=4\left(\gamma_{(12)}+2 \tau_{(12)}\right), \quad f_{7}^{U}=8 \gamma_{(12)}, \quad f_{8}^{U}=4 \gamma_{(12)}, \\
& f_{9}^{U}=16 \tau_{(12)}, \quad f_{10}^{U}=8\left(\gamma_{(12)}+2 \tau_{(12)}\right), \\
& f_{11}^{U}=4 \gamma_{(12)} \rho_{1}-2 \beta_{1} \gamma_{(12)}+8 \tau_{(12)}\left(\beta_{1}+4 \rho_{1}\right) \\
& f_{12}^{U}=4 \gamma_{(12)} \rho_{2}-2 \beta_{1} \gamma_{(22)}+8 \tau_{(12)}\left(\beta_{2}+4 \rho_{2}\right) \\
& f_{13}^{U}=4\left(\gamma_{(12)}+2 \tau_{(12)}\right)
\end{aligned}
$$

B.1.4. Field $A_{\mu}$ Right Side : Fields $V_{\mu}^{+}$and $V_{\mu}^{-}$

$$
\begin{aligned}
& f_{1}^{+}=-8 b_{3}\left(\gamma_{[13]}+i \gamma_{[14]}\right), \quad f_{2}^{+}=-4 \gamma_{[13]}, \quad f_{3}^{+}=-8 \gamma_{[13]}-2 i \triangle \gamma_{[14]} \\
& f_{4}^{+}=8 \beta_{3}\left(\gamma_{(13)}+i \gamma_{(14)}\right), \quad f_{5}^{+}=-\left(f_{3}^{+}\right)^{*}, \quad f_{6}^{+}=2 i \gamma_{[13]} \\
& f_{7}^{+}=4 \gamma_{[13]}, \quad f_{8}^{+}=-8 \gamma_{(13)}+2 i\left(\gamma_{(14)}+2 \tau_{(14)}\right), \quad f_{9}^{+}=-\left(f_{8}^{+}\right)^{*} \\
& f_{10}^{+}=2 i\left(\gamma_{(13)}+2 \tau_{(13)}\right), \quad f_{11}^{+}=4 i\left(\gamma_{(14)}+4 \tau_{(14)}\right), \quad f_{12}^{+}=4 i\left(\gamma_{(13)}+4 \tau_{(13)}\right), \\
& f_{13}^{+}=8 \rho_{3}\left(\gamma_{(13)}+I \gamma_{(14)}\right)+8\left(\beta_{3}+4 \rho_{3}\right)\left(\tau_{(13)}+i \tau_{(14)}\right)-2 \beta_{1} \gamma_{(33)} \\
& f_{14}^{+}=-f_{2}^{+}, \quad f_{15}^{+}=16\left(\gamma_{(13)}+2 \tau_{(13)}\right)
\end{aligned}
$$




\section{B.2. Field $U_{\mu}$}

\section{B.2.1. Left Side}

$$
\begin{aligned}
& \bar{b}_{1}=4\left(a_{2}+b_{(22)}\right), \quad \bar{b}_{2}=8 b_{2}, \quad \bar{b}_{3}=8 \beta_{2}, \\
& \bar{b}_{4}=4 c_{(12)}+\xi_{(12)} \\
& \bar{b}_{5}=4\left(b_{(22)}+c_{(22)}\right)+\xi_{(22)} \\
& \bar{b}_{6}=2\left(2 \rho_{2}-\beta_{2}\right), \quad \bar{b}_{7}=4\left(\beta_{2}+4 \rho_{2}\right)
\end{aligned}
$$

\section{B.2.2. Field $U_{\mu}$ Right Side : Field $A_{\mu}$}

$$
\begin{aligned}
& g_{1}^{A}=4 \gamma_{[12]} b_{1}, \quad g_{2}^{A}=4 \gamma_{[12]} b_{2}, \quad g_{3}^{A}=8 \gamma_{[12]}, \quad g_{4}^{A}=16\left(\gamma_{(12)}+\tau_{(12)}\right) \\
& g_{5}^{A}=4\left(\beta_{1} \gamma_{(12)}-\beta_{2} \gamma_{(11)}\right), \quad g_{6}^{A}=4 \gamma_{(12)}, \quad g_{7}^{A}=8 \gamma_{(12)} \quad g_{8}^{A}=4\left(\gamma_{(12)}+2 \tau_{(12)}\right), \\
& g_{9}^{A}=8\left(\gamma_{(12)}+2 \tau_{(12)}\right), \quad g_{10}^{A}=16 \tau_{(12)}, \quad g_{11}^{A}=4\left(\rho_{1} \gamma_{(12)}+2 \tau_{(12)}\left(\beta_{1}+4 \rho_{1}\right)\right)-2 \beta_{2} \gamma_{(11)} \\
& g_{12}^{A}=4\left(\rho_{2} \gamma_{(12)}+2 \tau_{(12)}\left(\beta_{2}+4 \rho_{2}\right)\right)-2 \beta_{2} \gamma_{(12)}
\end{aligned}
$$

\section{B.2.3. Field $U_{\mu}$ Right Side : Field $U_{\mu}$}

$$
\begin{aligned}
& g_{1}^{U}=4\left(\gamma_{(22)} \beta_{1}-\gamma_{(12)} \beta_{2}\right), \quad g_{2}^{U}=4 \gamma_{(22)}, \quad g_{3}^{U}=8\left(\gamma_{(22)}+2 \tau_{(22)}\right) \\
& g_{4}^{U}=4\left(\gamma_{(22)} \rho_{1}+2 \tau_{(22)}\left(\beta_{1}+4 \rho_{1}\right)\right)-2 \beta_{2} \gamma_{(12)} \\
& g_{5}^{U}=4\left(\gamma_{(22)} \rho_{1}+2 \tau_{(22)}\left(\beta_{2}+4 \rho_{2}\right)\right)-2 \beta_{2} \gamma_{(22)}
\end{aligned}
$$

\section{B.2.4. Field $U_{\mu}$ Right Side : Fields $V_{\mu}^{+}$and $V_{\mu}^{-}$}

$$
\begin{aligned}
& g_{1}^{+}=-8 b_{3}\left(\gamma_{[23]}+i \gamma_{[24]}\right), \quad g_{2}^{+}=2 i \gamma_{[24]}, \quad g_{3}^{+}=2 i \gamma_{[23]} \\
& g_{4}^{+}=4 \gamma_{[23]}, \quad g_{5}^{+}=8 \beta_{3}\left(\gamma_{(23)}+i \gamma_{(24)}\right)-4 \beta_{2} \gamma_{(33)}, \quad g_{6}^{+}=2 i\left(\gamma_{(24)}+2 \tau_{(24)}\right) \\
& g_{7}^{+}=-2 i\left(\gamma_{(23)}-2 \tau_{(23)}\right), \quad g_{8}^{+}=4 \gamma_{(23)} \\
& g_{9}^{+}=-4 i\left(\gamma_{(24)}+2 \tau_{(24)}\right), \quad g_{10}^{+}=4 i\left(\gamma_{(23)}+4 \tau_{(23)}\right) \\
& g_{11}^{+}=8 \rho_{3}\left(\gamma_{(23)}+i \gamma_{(24)}\right)+8\left(\beta_{3}+4 \rho_{3}\right)\left(\tau_{(23)}+i \tau_{(24)}\right)-2 \beta_{2} \gamma_{(33)} \\
& g_{12}^{+}=16\left(\gamma_{(23)}+2 \tau_{(23)}\right)
\end{aligned}
$$




\section{B.3. Field $V_{\mu}^{+}$}

\section{B.3.1. Left Side}

$$
\begin{aligned}
& \bar{c}_{1}=4\left(a_{3}+b_{(33)}\right), \quad \bar{c}_{2}=8 b_{3}, \quad \bar{c}_{3}=4\left(b_{(33)}+c_{(33)}\right)+\frac{1}{2} \xi_{(33)} \\
& \bar{c}_{4}=4\left(2 \rho_{3}-\beta_{3}\right), \quad \bar{c}_{5}=8\left(\beta_{3}+4 \rho_{3}\right)
\end{aligned}
$$

\section{B.3.2. Field $V_{\mu}^{+}$Right Side : Field $A_{\mu}$}

$$
\begin{aligned}
& h_{1}^{A}=4 b_{3}\left(\gamma_{[13]}+i \gamma_{[14]}\right), \quad h_{2}^{A}=4 \gamma_{[13]}, \quad h_{3}^{A}=8 \gamma_{[13]}+2 i \gamma_{[14]}, \\
& h_{4}^{A}=8 \gamma_{(13)}-2 i\left(\gamma_{(14)}-2 \tau_{(14)}\right), \quad h_{5}^{A}=2 i \gamma_{[13]} \\
& h_{6}^{A}=8 \beta_{3} i \gamma_{(14)}, \quad h_{7}^{A}=8 \gamma_{[13]}-2 i \gamma_{[14]} \\
& h_{8}^{A}=4 \gamma_{(13)}, \quad h_{9}^{A}=2 i\left(\gamma_{(13)}-2 \tau_{(13)}\right) \\
& h_{10}^{A}=-4 i\left(\gamma_{(14)}+2 \tau_{(14)}\right), \quad h_{11}^{A}=4 i\left(\gamma_{(13)}+4 \tau_{(13)}\right) \\
& h_{12}^{A}=4\left(\rho_{3}\left(\gamma_{(13)}+i \gamma_{(14)}\right)+\left(\beta_{3}+4 \rho_{3}\right)\left(\tau_{(13)}+i \tau_{(14)}\right)\right)-2 \beta_{3}\left(\gamma_{(13)}-i \gamma_{(14)}\right) \\
& h_{13}^{A}=16\left(\gamma_{(13)}+2 \tau_{(13)}\right)
\end{aligned}
$$

\section{B.3.3. Field $V_{\mu}^{+}$Right Side : Field $U_{\mu}$}

$$
\begin{aligned}
& h_{1}^{U}=4 b_{3}\left(\gamma_{[13]}+i \gamma_{[14]}\right), \quad h_{2}^{U}=2 i \gamma_{[24]}, \quad h_{3}^{U}=-2 i \gamma_{[23]}, \quad h_{4}^{U}=4 \gamma_{[23]} \\
& h_{5}^{U}=8 \beta_{3} i \gamma_{(24)}, \quad h_{6}^{U}=2 i\left(\gamma_{(24)}-2 \tau_{(24)}\right), \quad h_{7}^{U}=2 i\left(\gamma_{(23)}+2 \tau_{(23)}\right), \\
& h_{8}^{U}=4 \gamma_{(23)}, \quad h_{9}^{U}=-4 i\left(\gamma_{(24)}+4 \tau_{(24)}\right), \quad h_{10}^{U}=4 i\left(\gamma_{(23)}+4 \tau_{(23)}\right) \\
& h_{11}^{U}=4\left(\rho_{3}\left(\gamma_{(13)}+i \gamma_{(14)}\right)+\left(\beta_{3}+4 \rho_{3}\right)\left(\tau_{(13)}+i \tau_{(14)}\right)\right)-2 \beta_{3}\left(\gamma_{(23)}-i \gamma_{(24)}\right) \\
& h_{12}^{U}=16\left(\gamma_{(23)}+2 \tau_{(23)}\right)
\end{aligned}
$$


B.3.4. Field $V_{\mu}^{+}$Right Side : Field $V_{\mu}^{-}$

$$
\begin{aligned}
& h_{1}^{-}=-4 i\left(b_{1} \gamma_{[34]}+\beta_{1} \gamma_{(34)}\right), \quad h_{2}^{-}=-4 i\left(b_{2} \gamma_{[34]}+\beta_{2} \gamma_{(34)}\right) \\
& h_{3}^{-}=8 i \gamma_{[34]}, \quad h_{4}^{-}=4 \gamma_{(33)} \beta_{1}-4 \beta_{3}\left(\gamma_{(13)}-i \gamma_{(14)}\right), \\
& h_{5}^{-}=4 \gamma_{(33)} \beta_{2}-4 \beta_{3}\left(\gamma_{(23)}-i \gamma_{(24)}\right) \\
& h_{6}^{-}=4 \gamma_{(33)}, \quad h_{7}^{-}=-8 i \gamma_{[34]}, \quad h_{8}^{-}=32 i\left(\gamma_{[34]}+2 \tau_{(34)}\right), \\
& h_{9}^{-}=8 \gamma_{(33)} \rho_{1}+4\left(\beta_{1}+4 \rho_{1}\right) \tau_{(33)}-2 \beta_{3}\left(\gamma_{(13)}-i \gamma_{(14)}\right), \\
& h_{10}^{-}=8 \gamma_{(33)} \rho_{1}+4\left(\beta_{2}+4 \rho_{2}\right) \tau_{(33)}-2 \beta_{3}\left(\gamma_{(23)}-i \gamma_{(24)}\right) \\
& h_{11}^{-}=8 \tau_{(33)}, \quad h_{12}^{-}=8 i \gamma_{[34]}, \quad h_{13}^{-}=8 i \gamma_{(34)}, \\
& h_{14}^{-}=16\left(\gamma_{(33)}+2 \tau_{(33)}\right),
\end{aligned}
$$




\section{References}

[1] J. C. Maxwell, London Edinburgh Dublin Phys. Mag and J.Sc. Series IV, 21, 161 (196)

[2] J. Chauca, R.Doria, I. Soares, JAP Vol 10 N 1 2605(2015); JAP, 13(1), 4535 (2017)

[3] N. Bohr, Philos. Mag. 26 (1913) 1; Philos. Mag. 26 (1913) 476

[4] Sin-Itiro Tomonaga, "The Story of Spin", Chicago University Press, 1998.

[5] A. Compton, The magnetic electron, Jour. Franklin Inst. 192:2, (1921) 145

[6] W. Gerlach; O. Stern, "Der experimentelle Nachweis der Richtungsquantelung im Magnetfeld". Zeitschrift für Physik. 9: 349-352(1922); W. Gerlach; O. Stern. "Das magnetische Moment des Silberatoms". Zeitschrift für Physik. 9: 353-355; Gerlach, W.; Stern, O. (1922). "Der experimentelle Nachweis des magnetischen Moments des Silberatoms". Zeitschrift für Physik. 8: 110-111.

[7] S. Goudsmith and R. Kronig, Naturwissenschaffen 13(1925) 90; S. Goudsmith and G. E. Uhlenbeck, Physica (Utrecht) 5 (1925) 266; S. Goudsmit and G. E. Uhlenbeck, Spinning electrons and the structure of spectra, Nature 117 (1926) 264; G. E. Uhlenbeck, Physics Today, June 1976, p. 43.

[8] L. Thomas, The motion of the spinning electron, Nature 117 (1926) 514

[9] W. Heisenberg, Quantum-theoretical re-interpretation of kinematic and mechanical relations, Zeitschrift für Physik $33(1925) 879$.

[10] W. Heisenberg, P. Jordan, Anwendung Der Quantenmechanik Auf Das Problem Der Anomalen Zeemaneffekte, Zeit.f.Physik 37 (1926) 263

[11] E. Schrödinger, An ondulatory theory of the mechanics of atoms and molecules, Phys. Rev. 28 (1926) 1049.

[12] W. Pauli Zer Quatermechanik des magnetischen Elektrons, Zeitschrift für Physik 43 (1927) 601.

[13] P.A.M Dirac The quantum theory of the electron-I, The quantum theory of the electron-II Proc.R.Soc.Lond. A 117 (1928) 610; Proc.R.Soc.Lond.A 118 (1928), 351.

[14] E. Cartan, "Theory of Spinors", Dover Publications Inc., New York, 1981.

[15] V. Weisskopf, Physics in the twentieth century, Select essays (1972)

[16] K. M. Case Phys. Rev. 94, 1442 (1954) C. Fronsdal, Nuovo Cimento Suppl. 9, 416 (1958) J. Schwinger, Particles, Sources, and Fields (Addison-Wesley, Reading, MA, 1970). L. P. S. Singh and C. R. Hagen, Phys. Rev. D9, 898 (1974); 9, 910 (1974).

[17] S. Weinberg, Lectures on Electromagnetism Particles and Quantum Field Theory, Proceedings of the Summer Institute, Brandeis University, 1970, edited by S. Defer (MIT Preas, Cambridge, MA, 1970), vol I.

[18] S. Ferrara, M. Porrati and V. Teledgi, Phis. Rev. D46, 3529 (1992).

[19] P. Kusch and H. Foley, The magnetic moment of the electron, Phys. Review, 74 (3): 250, (1948)

[20] D. Nagle, R. Julian and S. Zacharias, Phys. Review 72 (1947) 1 
[21] J. Schwinger, Phys. Rev. 76 (1949) 6

[22] K. A. Olive at al. Review of Particle Physics. Chin. Phys. C 38 (2014); G. W. Bennet et al, improved limit on the muon dipole moment, Phys. Review D 80 (2009) 5

[23] D. Lee and C. N. Yang. Theory of charged vector mesons interacting with the electromagnetic field. Phys. Rev., 128:885-898, Oct 1962, Abdus Salam. Renormalizable electrodynamics of vector mesons. Phys. Rev., 130:1287-1290, May 1963, K. H. Tzou. Il Nuovo Cimento, 33:286, 1964; Abdus Salam and Robert Delbourgo. Renormalizable electrodynamics of scalar and vector mesons. ii. Phys. Rev., 135:B1398-B1427, Sep 1964.

[24] L.Fonda, G.Chirardi, Symmetry Principles in Physics, Chapter III

[25] N. Ramsay, Reports on progress in physics, 45(1) (1982),95

[26] E. Wigner, On unitary representation of the inhomogeneus Lorentz Group, Annals of Mathematics, 149-204(1939)

[27] J. Helayel, www.professorglobal.com.br

[28] E.Noether, Invariante Variations Probleme, Math.-Phys Klasse (1918) 235

[29] I.P. Ivanov et al, Phys. Rev. Lett. 124 (2020) 192001; T. Fujita, M.B.A. Jalil, S.G. Tan, S. Murakami, Gauge Fields in spintronics, J. Appl. Phys. 110, 121301 (2011); H. Ohanian, Quantum Measurements, American Journal of Physics 85, 5, (2016) "And what is spin", Am. J. Phys. 54,6 (1986)

[30] Y. Aharanov and D. Bohm, Significance of the electromagnetic potentials of the quantum theory, Phys. Rev. 115 (1959) 485

[31] K.Rith and A.Schafer, The Mistery of Nuclear Spin, Scientific American, July (1999) 